\title{
Symplectic geometry and Floer homology
}

\section{François Laudenbach}

In memory of Laurent Schwartz whose charm was so very influential. I owe it to him to have become a mathematician.

Summary. Here are the revised notes for lectures held at the 13th Brazilian Topology Meeting in Belo Horizonte (July 2002). The purpose is to give an introduction to symplectic Floer homology and, in a simple case, a sketch of proof of the Arnold conjecture. This conjecture gives a lower bound for the number of fixed points of a Hamiltonian diffeomorphism in terms of the sum of the Betti numbers. Floer theory is a sort of infinite dimensional Morse theory on a loop space. The Morse index is replaced by the Maslov-Conley-Zehnder index. Some results about the Maslov cycle in the linear symplectic group are gathered in an appendix.

\section{A conjecture by V.I. Arnold}

Conjecture [4]. Actually the conjecture goes back to Arnold's note [1]. Here is the statement:

Let $\left(M^{2 n}, \omega\right)$ be a symplectic closed manifold. Let $\varphi_{1}$ be a Hamiltonian diffeomorphism of $M$. Then $\varphi_{1}$ has at least as many fixed points as a smooth function has critical points:

$$
\# \operatorname{Fix} \varphi_{1} \geq \inf _{f \in C^{\infty}(M, \mathbb{R})} \# \operatorname{crit} f
$$

If we think only of the generic case, it is natural to look at Morse functions. Remembering the Morse inequalities, one can extract a weaker form of the conjecture. 
Weak conjecture. For a generic Hamiltonian diffeomorphism, the number of fixed points is at least the sum of the Betti numbers:

$$
\# \operatorname{Fix} \varphi_{1} \geq \sum_{i} \operatorname{rank} H_{i}(M, \mathbb{Z})
$$

Conley and Zehnder proved the strong conjecture for $M=T^{2 n}$ in [14]. A particular case of the weak Arnold conjecture - when $(M, \omega)$ is called monotone - has been proven by Andreas Floer [24]. Since that time other cases of the weak conjecture have been proven; the most general case has been solved by K. Fukaya and K. Ono [25], and also by G. Liu and G. Tian [35]. But as stated above the Arnold conjecture is still an open question, in particular in the nongeneric case (except for $T^{2 n}$ ). In this mini-course we would like to explain a very simple case of the weak conjecture where Floer's ideas apply. First we recall a little from symplectic geometry as well as the prehistory of the subject which begins with Poincaré's last geometric theorem.

\subsection{Basic definitions in symplectic geometry}

A symplectic structure on a manifold $M$ is given by a 2 -form $\omega \in \Omega^{2}(M)$, which satisfies the two following conditions:

1) $d \omega=0$,

2) for every $x \in M, \omega(x): T_{x} M \times T_{x} M \rightarrow \mathbb{R}$ is bilinear, skew-symmetric and nondegenerate.

The determinant of a skew-symmetric $n \times n$ matrix vanishes when $n$ is odd. Then condition 2 forces the dimension of $M$ to be even, $\operatorname{dim} M=2 n$, and the non-degeneracy condition just says that $\omega^{n}:=\omega \wedge \ldots \wedge \omega$ is a volume form on $M$. In particular $M$ is oriented.

Example. $M=\mathbb{R}^{2 n}$ with coordinates $\left(p_{i}, q_{i}\right), \omega_{0}=\sum d p_{i} \wedge d q_{i}$ and $\omega_{0}^{n}=n !\left(d p_{1} \wedge d q_{1} \wedge \ldots \wedge d p_{n} \wedge d q_{n}\right)$.

Darboux's Theorem. Any $2 n$-dimensional symplectic manifold is locally isomorphic to $\left(\mathbb{R}^{2 n}, \omega_{0}\right)$.

Definition 1.1. Thinking of $\mathbb{R}^{2 n}$ as the cotangent space $T^{*} \mathbb{R}^{n}=\mathbb{R}^{n} \times \mathbb{R}^{n}$, where the $q_{i}$ 's are the coordinates on the basis and the $p_{i}$ 's the coordinates on the fibre, the standard Liouville form is $\lambda_{0}=\sum p_{i} d q_{i}$ and $d \lambda_{0}=\omega_{0}$.

Theorem 1.2. The Liouville form $\lambda_{0}$ is "natural" in the following sense. Let $\varphi: U \rightarrow V$ be a (local) diffeomorphism of $\mathbb{R}^{n}, Q=\varphi(q)$. Let $\Phi: U \times \mathbb{R}^{n *} \rightarrow$ $V \times \mathbb{R}^{n *}$ be its cotangent extension:

$$
\Phi(q, p):=\left(\varphi(q),{ }^{t}(D \varphi(q))^{-1}\right) .
$$

Then $\Phi^{*}\left(\sum P_{i} d Q_{i}\right)=\sum p_{i} d q_{i}={ }^{t} p . d q$. 
Proof. $\Phi^{*}\left({ }^{t} P \cdot d Q\right)={ }^{t} p \cdot D \varphi(q)^{-1} \cdot D \varphi(q) \cdot d q$.

Corollary 1.3. Let $M=T^{*} N$ be the cotangent space of $N$. There exists a 1-form, called the canonical Liouville form $\lambda$, which is given in coordinates as $p d q$. Its differential $\omega=d \lambda$ is closed and is given locally by $d p \wedge d q$. So $\omega$ is a symplectic form.

Other examples: $T^{2 n}, P^{n}(\mathbb{C})$, the orientable surfaces equipped with an area form.

Definition 1.4. With a smooth function $H: \mathbb{R} \times \mathbb{R}^{2 n} \rightarrow \mathbb{R},(t, p, q) \mapsto H(t, p, q)$ $=H_{t}(p, q)$, called a Hamiltonian (function), one can associate a Hamiltonian differential system:

$$
\left\{\begin{array}{l}
\dot{p}=-\frac{\partial H_{t}}{\partial q} \\
\dot{q}=+\frac{\partial H_{t}}{\partial p}
\end{array}\right.
$$

Example. With the Hamiltonian $H=\frac{p^{2}}{2}+V(q)$ the following system is associated:

$$
\left\{\begin{array}{l}
\dot{p}=-\nabla V \\
\dot{q}=p
\end{array}\right.
$$

When $H$ is time independent, $H(p(t), q(t))$ is constant along any solution of the system.

Notation 1.5. Let us identify $\mathbb{R}^{2 n}$ and $\mathbb{C}^{n}=\left\{p_{1}+i q_{1}, \ldots\right\}$. If $J$ denotes the operator of multiplication by $i$ in each complex factor, the Hamiltonian system from definition 1.4 reads:

$$
\dot{x}=J \nabla H_{t}(x) \text {. }
$$

Definition 1.6. On a symplectic manifold $(M, \omega)$ let $H: \mathbb{R} \times M \rightarrow \mathbb{R}$ be a smooth Hamiltonian function. We denote $X_{H_{t}}$ the Hamiltonian vector field $\omega$-dual of $d H_{t}$ :

$$
i\left(X_{H_{t}}\right) \omega=-d H_{t} .
$$

For each $t, X_{t}$ is also called the symplectic gradient of the function $H_{t}$ and reads $X_{t}=\nabla_{\omega} H_{t}$.

The sign - is due to the usual convention in classical mechanics. When $(M, \omega)=$ $\left(\mathbb{R}^{2 n}, \omega_{0}\right)$, then $\nabla_{\omega}=J \nabla$.

Proposition 1.7. A time dependent vector field $X_{t}$ is Hamiltonian iff it satisfies the two following conditions:

1) $\varphi_{t}^{*} \omega=\omega$ where $\varphi_{t}$ denotes its flow,

2) for any closed curve $c, \int_{c} i\left(X_{t}\right) \omega=0$. 
Proof. $\Rightarrow 1) \frac{d}{d t} \varphi_{t}^{*} \omega=\varphi_{t}^{*}\left(L_{X_{t}} \omega\right)=\varphi_{t}^{*}\left(i\left(X_{t}\right) d \omega+d i\left(X_{t}\right) \omega\right)=\varphi_{t}^{*}\left(-d d H_{t}\right)=$ 0 .

2) $\int_{c} d H_{t}=0$.

$\Leftarrow$ By the above calculation the first condition implies that the 1-form $i\left(X_{t}\right) d \omega$

is closed. According to the second condition it is exact.

Definition 1.8. The time 1 map $\varphi_{1}$ from a Hamiltonian flow is said a Hamiltonian diffeomorphism. $\operatorname{Ham}(M)$ is the set of Hamiltonian diffeomorphisms.

Exercise. $\operatorname{Ham}(M)$ is a group.

Lemma 1.9. The same $\varphi_{1}$ can be obtained from a Hamiltonian function which is 1-periodic in time.

Proof. Let $H_{t}$ be a Hamiltonian yielding the flow $\varphi_{t}$. Then we have

$$
\frac{d}{d t} \varphi_{\alpha(t)}=\dot{\varphi}_{\alpha(t)} \alpha^{\prime}(t)=X_{H_{t}}\left(\varphi_{\alpha(t)}\right) \alpha^{\prime}(t)=X_{\alpha^{\prime}(t) H_{t}}\left(\varphi_{\alpha(t)}\right) .
$$

Then $K_{t}:=\alpha^{\prime}(t) H_{t}$ is a Hamiltonian for the isotopy $\left\{\varphi_{\alpha(t)}\right\}$. If $\alpha:[0,1] \rightarrow$ $[0,1]$ we have $\varphi_{\alpha(1)}=\varphi_{1}$. Moreover if $\alpha(t)=i, i=0,1$, for $t$ close to $i, K_{t}=0$ near 0 and 1 and then it can be extended periodically.

Note. In the rest of this text any Hamiltonian $H$ under consideration will be 1-periodic in time, ie: $H: S^{1} \times M \rightarrow \mathbb{R}$, where $S^{1}=\mathbb{R} / \mathbb{Z}$. If $\varphi_{t}$ denotes its flow, we have the following $1-1$ correspondence:

$$
\text { Fix } \varphi_{1} \Longleftrightarrow\left\{1-\text { periodic orbits of } X_{H_{t}}\right\} \text {. }
$$

\subsection{The Poincaré - Birkhoff Theorem}

Theorem 1.10. Let $f$ be an area preserving homeomorphism of the annulus $A:=S^{1} \times[-1,+1]$ which satisfies the twist condition. Then $f$ has at least 2 fixed points. [7]

Moreover there are infinitely many periodic points, all geometrically distinct [12].

The universal cover is $\widetilde{A}=\mathbb{R} \times[-1,+1]$. Let $(p, q)$ be the coordinates in this product. On $A, p$ becomes an angular coordinate. The area form is $\omega=d p \wedge d q$. A lift $\tilde{f}$ to $\widetilde{A}$ reads

$$
\begin{aligned}
& (p, q) \mapsto\left(p^{\prime}, q^{\prime}\right) \\
& p^{\prime}=p+a(p, q) \\
& q^{\prime}=q+b(p, q) .
\end{aligned}
$$

where $a$ and $b$ are two functions 1-periodic in $p$, such that $b$ vanishes for $q= \pm 1$ : 
Definition 1.11. The homeomorphism $f$ satisfies the twist condition if there is some lift $\tilde{f}$ to $\widetilde{A}$ with $a(p,+1)>0$ and $a(p,-1)<0$.

We will give a proof in two particular cases. The first one is very particular, but it shows clearly how the fixed points problem is related to the critical points problem of functions. The second one could be called the Poincaré case. The idea of generating functions comes to play there.

\section{Proof.}

1) The very particular case. Here $X=\nabla_{\omega} h$ where $h: A \rightarrow \mathbb{R}$ is a function which is independent of time and constant along each component of the boundary, with a (usual) gradient pointing inside the annulus. If $\vec{n}$ is an outgoing vector at each point of $\partial A$, we have $\omega(X, \vec{n})>0$ and then, if $f$ is the time 1 map of the flow of $X$ the twist condition is satisfied.

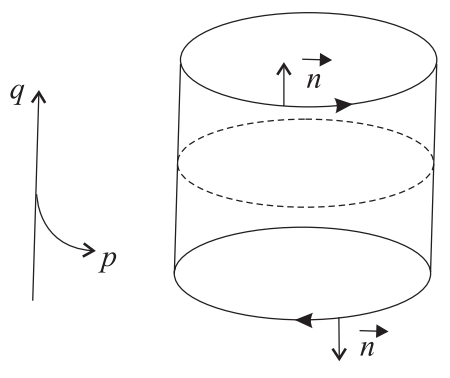

Figure 1

In this situation we have:

$$
\text { crit } h \subset \operatorname{Fix} f \text {. }
$$

We have to prove: \#crit $h \geq 2$. Certainly there is some maximum which is not on the boundary since the gradient of $h$ points in. So it is a first critical point lying in the interior of $A$. Let us assume there is only one maximum. The second critical point will be obtained by a minimax argument.

Let $\mathcal{T}=\left\{\alpha:[-1,+1] \rightarrow S^{1} \times[-1,+1] \mid \alpha\right.$ is $\left.C^{0}, \alpha( \pm 1) \in S^{1} \times\{ \pm 1\}\right\}$. By definition the minimax value is

$$
c:=\inf _{\alpha \in \mathcal{T}}\left(\max _{t} h \circ \alpha(t)\right) .
$$

Due to the boundary condition and because the absolute maximum is unique, we have:

$$
\max \left(h\left(S^{1} \times\{ \pm 1\}\right)\right)<c<\max h .
$$

The existence of the second critical point follows by the following lemma. 


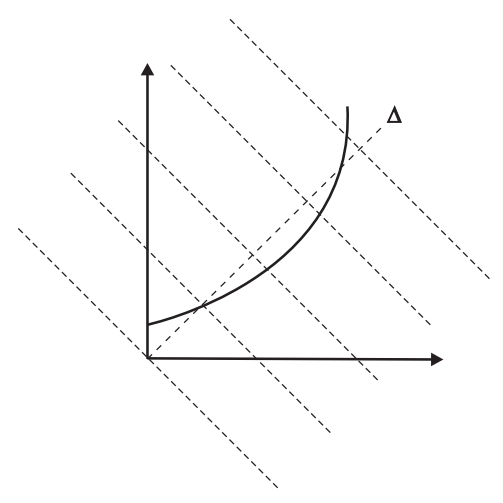

Figure 2

Lemma 1.12. The minimax value $c$ is a critical value.

Proof. If not, for some $\varepsilon>0, h^{-1}([c-\varepsilon, c+\varepsilon])$ is diffeomorphic to $h^{-1}(c) \times$ $[c-\varepsilon, c+\varepsilon]$ and any path from $\mathcal{T}$ lying in $\left.\left.h^{-1}(]-\infty, c+\varepsilon\right]\right)$ can be pushed by a homotopy with fixed endpoints into $\left.\left.h^{-1}(]-\infty, c-\varepsilon\right]\right)$ which contradicts the definition of $c$.

2) The Poincaré case. Here $f$ is assumed $C^{1}$-close to Identity (without lying on a 1-parameter group).

Let $\tilde{f}$ be a lift of $f$ as in definition 1.11. We look at the graph $G(\tilde{f})$ in $\widetilde{A} \times \widetilde{A}$ endowed with the symplectic form $\omega \ominus \omega:=d p^{\prime} \wedge d q^{\prime}-d p \wedge d q$. The area preserving property of $f$ is equivalent to $G(\tilde{f})$ being a Lagrangian submanifold (ie: $\omega \ominus \omega$ induces the null 2 -form on $G(\tilde{f})$ ). Now we can make precise the closeness of $f$ to $I d$ :

$$
\text { (*) } \quad \operatorname{det}(D \tilde{f}+I d) \neq 0 .
$$

This condition amounts to the transversality of $G(\tilde{f})$ to the anti-diagonal foliation.

Lemma 1.13. Let $\Theta: \widetilde{A} \times \widetilde{A} \rightarrow T^{*} \widetilde{A} \cong \mathbb{R}^{2} \times \widetilde{A}$ be defined by

$$
\left(p, q, p^{\prime}, q^{\prime}\right) \mapsto P_{1}=q-q^{\prime}, P_{2}=p^{\prime}-p, Q_{1}=\frac{p+p^{\prime}}{2}, Q_{2}=\frac{q+q^{\prime}}{2}
$$

Then:

1) $\Theta$ is a symplectic embedding, 
2) the image of the diagonal $\Delta$ is $\{0\} \times \widetilde{A}$,

3) $\Theta$ is equivariant with respect to the actions of $\mathbb{Z}$ given by $\left(p, q, p^{\prime}, q^{\prime}\right) \mapsto$ $\left(p+1, q, p^{\prime}+1, q^{\prime}\right)$ and $\left(P_{1}, P_{2}, Q_{1}, Q_{2}\right) \mapsto\left(P_{1}, P_{2}, Q_{1}+1, Q_{2}\right)$.

Proof. By calculation we get $d P_{1} \wedge d Q_{1}+d P_{2} \wedge d Q_{2}=d p^{\prime} \wedge d q^{\prime}-d p \wedge d q$. The other points are obvious.

On $\Theta(G(\tilde{f}))$ we have:

(1) $d P_{1} \wedge d Q_{1}+d P_{2} \wedge d Q_{2}=0$

(2) $P_{1}=P_{1}\left(Q_{1}, Q_{2}\right), P_{2}=P_{2}\left(Q_{1}, Q_{2}\right)$.

Condition (2) means that $\Theta(G(\tilde{f}))$ is the graph of some 1-form

$$
\alpha\left(Q_{1}, Q_{2}\right)=P_{1}\left(Q_{1}, Q_{2}\right) d Q_{1}+P_{2}\left(Q_{1}, Q_{2}\right) d Q_{2},
$$

and condition (1) means that $d \alpha=0$. By the so-called Poincaré Lemma $\alpha=d \widetilde{S}$ for some function $\widetilde{S}: \widetilde{A} \rightarrow \mathbb{R}$ and:

$$
\left\{\begin{array}{l}
q-q^{\prime}=\partial_{1} \widetilde{S} \\
p^{\prime}-p=\partial_{2} \widetilde{S}
\end{array}\right.
$$

These last formulae make clear the following equivalence:

$$
(p, q) \in \operatorname{Fix} \tilde{f} \Longleftrightarrow(p, q) \in \operatorname{crit} \widetilde{S} .
$$

If $q=q^{\prime}=-1$, then $Q_{2}=\frac{q+q^{\prime}}{2}=-1, P_{1}=0$ and $P_{2}<0$ according to the twist condition.

If $q=q^{\prime}=+1$, then $Q_{2}=+1, P_{1}=0$ and $P_{2}>0$. Therefore $\widetilde{S}$ is constant along each boundary component of $\widetilde{A}$ and the gradient points out.

Lemma 1.14. $\widetilde{S}: \widetilde{A} \rightarrow \mathbb{R}$ is 1-periodic in $Q_{1}$.

Proof. The graph $G(\tilde{f})$ is $\mathbb{Z}$-invariant. Then according to 3 ) from lemma 1.13 the differential of $\widetilde{S}$ is 1-periodic in $Q_{1}$. So it is sufficient to check the periodicity of $\widetilde{S}$ along $\left\{Q_{2}=1\right\}$. But there $\widetilde{S}$ is constant, a fortiori 1-periodic.

As a consequence $\widetilde{S}$ descends to the annulus $A=S^{1} \times[-1,+1]$ hence defines a function $S: A \rightarrow \mathbb{R}$. Knowing the position of its gradient along the boundary and using the minimax argument (lemma 1.12), we see that $S$ has at least two critical points. It finishes the proof that $f$ has at least two fixed points.

Remark 1.15. A function $S$ as above is called a generating function of the symplectic diffeomorphism $f$. The same idea does not work directly when $f$ is not close enough to the Identity. But in [30] L. Hörmander introduced a generalization, called generating phase or generating family in Weinstein's terminology. With this tool M. Chaperon succeeded in giving a simple proof of the Arnold conjecture for the $2 n$-torus, previously proven by C. Conley and E. Zehnder [14]. As V.I. Arnold noticed in Appendix 9 from [1], the case $n=1$ includes the $C^{1}$ - Poincaré-Birkhoff theorem. 


\section{Symplectic action on loops}

\subsection{Action functional in case of $\left(\mathbb{R}^{2 n}, \omega_{0}=d p \wedge d q\right)$}

Let $H \in C^{\infty}\left(S^{1} \times \mathbb{R}^{2 n}, \mathbb{R}\right)$ be a 1-periodic time dependent Hamiltonian and let $H_{t}$ be its restriction to $\{t\} \times \mathbb{R}^{2 n}$. Denote $\Lambda:=C^{\infty}\left(S^{1}, \mathbb{R}^{2 n}\right)$. For $x \in \Lambda$, $x(t)=(p(t), q(t))$, we define the action functional by the following formula:

$$
\Phi_{H}(x)=-\int_{S^{1}} x^{*}[p d q-H d t]=-\int_{S^{1}}[p(t) \dot{q}(t)-H(t, x(t))] d t .
$$

If $\delta x$ is a tangent vector to $\Lambda$ at $x$ ( $i e$ a vector field in $\mathbb{R}^{2 n}$ along $x$ ), the differential of $\Phi_{H}$ is well defined in the direction of $\delta x$ and we have:

$$
d \Phi_{H}(x) . \delta x=-\int_{S^{1}}\left[\dot{q} \delta p+p \dot{\delta q}-d H_{t}(x) . \delta x\right] d t
$$

After integration by parts we get:

$$
\begin{aligned}
d \Phi_{H}(x) . \delta x & =-\int_{S^{1}}\left[\dot{q} \delta p-\dot{p} \delta q-d H_{t} . \delta x\right] d t \\
& =-\int_{S^{1}}\left[\omega_{0}(\delta x(t), \dot{x}(t))-d H_{t}(x) . \delta x\right] d t \\
& =+\int_{S^{1}}\left[\omega_{0}(\dot{x}, \delta x)+d H_{t}(x) \cdot \delta x\right] d t .
\end{aligned}
$$

This computation is basic in the calculus of variations and yields the following proposition:

Proposition 2.1. The loop $x$ is a critical point of $\Phi_{H} \Leftrightarrow \omega_{0}(\dot{x}, \cdot)+d H_{t}(x)=$ $0 \Leftrightarrow \dot{x}=X_{H_{t}}(x) \Leftrightarrow x$ is a 1-periodic solution of the Hamiltonian system from definition 1.4 .

\subsection{Case of an autonomous $C^{2}$-small Hamiltonian}

Lemma 2.2. Let $H: \mathbb{R}^{2 n} \rightarrow \mathbb{R}$ be an autonomous Hamiltonian function satisfying $\|d \nabla H(x)\|<2 \pi$. Then any 1-periodic solution of the Hamiltonian system is constant and coincides with a critical point of $H$.

Proof. The $n$-th Fourier coefficient of $x$ is $c_{n}(x)=\int_{0}^{1} e^{-2 i \pi n t} x(t) d t$ and $c_{n}(\dot{x})=$ $-2 i \pi n c_{n}(x)$. Since $c_{0}(\dot{x})=0$ from Parseval's formula we have:

$$
\|\dot{x}\|_{L^{2}} \leq \frac{1}{2 \pi}\|\ddot{x}\|_{L^{2}} .
$$

But $\ddot{x}=J d \nabla H(x) . \dot{x}$ implies $\|\ddot{x}\|_{\infty}<2 \pi\|\dot{x}\|_{\infty}$, which leads to a contradiction if $x$ is not constant. 
Corollary 2.3. Let $M$ be a closed symplectic manifold and $H: M \rightarrow \mathbb{R}$ an autonomous Hamiltonian. If $H$ is $C^{2}$-small enough, any 1-periodic solution of $\dot{x}=X_{H}(x)$ is constant.

Proof. Let us equip $M$ with a finite atlas of compact symplectic charts. This means a finite covering of $M$ with the interiors of compact domains $K_{i}$ on the neigbourhood of which symplectic charts are defined. If $H$ is $C^{1}$-small, every solution of $\dot{x}=X_{H}(x)$ has a small diameter and then it remains in one of the $K_{i}$ 's. Here the $C^{2}$ norm is equivalent to the Euclidian norm of $\ddot{x}$ in the symplectic coordinates of $K_{i}$ and the above lemma applies.

There are two consequences of the previous discussion.

1) If our goal is to estimate from below \#Per $\left(X_{H_{t}}\right)$, the number of 1-periodic solutions, independently of $H$, we see that we have to take only the contractible orbits into account.

2) In the case of Lemma 2.2 we have $\operatorname{Per}\left(X_{H}\right)=$ crit $H$ showing how the Morse theory comes into the problem of periodic orbits.

\subsection{Action functional in the case of the $2 n$-torus}

Let $\pi: \mathbb{R}^{2 n} \rightarrow T^{2 n}$ be the universal cover. Let us denote $\Lambda=C_{c}^{\infty}\left(S^{1}, T^{2 n}\right)$, the space of smooth contractible loops. For $x \in \Lambda$ we have $u: D^{2} \rightarrow T^{2 n}$, which is unique up to homotopy relatively to $S^{1}$ (because the 2nd homotopy group of $T^{2 n}$ is trivial), and $\tilde{x}: S^{1} \rightarrow \mathbb{R}^{2 n}$ such that $x$ is the restriction of $u$ to $S^{1}$ and $x=\pi \circ \tilde{x}$. Given a Hamiltonian $H$ 1-periodic in time, the action integral is now defined by:

$$
\Phi_{H}(x)=-\int_{S^{1}} \tilde{x}^{*}(p d q)+\int_{S^{1}} H(t, x) d t .
$$

By the Stokes formula we also have:

$$
\Phi_{H}(x)=-\int_{D^{2}} u^{*} \omega_{0}+\int_{S^{1}} H(t, x) d t
$$

where $\omega_{0}$ denotes the standard symplectic structure on $T^{2 n}$.

\subsection{Homotopic assumption}

In order to define the action integral for a more general symplectic manifold $(M, \omega)$ we will for simplicity make the following very strong assumption:

$$
<\omega, \pi_{2}(M)>=0
$$

$i e$ : for any $V: S^{2} \rightarrow M, \int_{S^{2}} v^{*} \omega=0$. Of course this assumption is much stronger than monotonicity, the assumption made in Floer's work. If $\Lambda$ still 
denotes the space of smooth contractible loops of $M$, for $x \in \Lambda$ the following integral is well defined:

$$
\Phi_{H}(x)=-\int_{D^{2}} u^{*} \omega+\int_{S^{1}} H(t, x) d t
$$

where $u: D^{2} \rightarrow M$ extends $x: S^{1} \rightarrow M$. The usual calculus of variations yields:

$$
x \in \operatorname{crit} \Phi_{H} \Longleftrightarrow x \text { is a } 1 \text {-periodic solution of } \dot{x}=X_{H_{t}} .
$$

In the sequel we will try to make a Morse theory for the action functional even though it is defined on an infinite dimensional space.

As a side-remark we observe that, when $\left\langle\omega, \pi_{2}(M)>\neq 0\right.$, the action functional becomes a multivalued function. Indeed for a given $x: S^{1} \rightarrow M$ there are different $u: D^{2} \rightarrow M$ providing different integrals $\int_{D^{2}} u^{*} \omega$. Only the differential of $\Phi_{H}$ is well defined. Hence the problem of counting the critical points deals with Morse-Novikov theory which is an analogue of Morse theory for closed 1-forms instead of real functions ([38], [28]). Actually there are other difficulties that appear simultaneously when the above homotopic assumption is not made, like the loss of compactness as it is stated in section 4 .

\section{5 $\quad L^{2}$-gradient of the action in the case of the standard torus}

First let us recall $J$, the complex structure on $\mathbb{C}^{n}$, and its relation with the symplectic structure $\omega_{0}$ and the Euclidean product. For $\xi, \eta \in \mathbb{C}^{n}$,

$$
\omega_{0}(\xi, \eta)=-<\xi, J \eta>
$$

Here $<,>$ stands for the Euclidean scalar product on $\mathbb{R}^{2 n}$. Now if $x$ is a smooth loop in $T^{2 n}$ and $\delta x$ a variation of $x$ we have:

$$
\begin{aligned}
d \Phi_{H}(x) . \delta x & =\int \omega_{0}(\dot{x}, \delta x)-\omega_{0}\left(X_{H}(t, x), \delta x\right) d t \\
& =\int\left(-<\dot{x}, J \delta x>+<X_{H}(t, x), J \delta x>\right) d t \\
& =\int_{S^{1}}<J \dot{x}+\nabla H, \delta x>d t \\
& =<J \dot{x}+\nabla H(t, x), \delta x>_{L^{2}} .
\end{aligned}
$$

Therefore the $L^{2}$-gradient of $\Phi_{H}$ is defined at $x \in \Lambda$ by:

$$
\operatorname{grad} \Phi_{H}(x)=J \dot{x}+\nabla H(t, x),
$$

which is a vector field tangent to $T^{2 n}$ along the loop $x$. 
Remark 2.4. One can check that the Cauchy problem associated with this gradient on $\Lambda$ is ill-posed. In order to correct this fact the very seminal idea of Floer is to look at the gradient lines globally. As in Morse theory one looks at descending gradient lines. Such a line in $\Lambda$ is a family of loops

$$
\begin{aligned}
u: & \mathbb{R} \times S^{1} \rightarrow T^{2 n} \\
& (s, t) \mapsto u(s, t)
\end{aligned}
$$

which is a solution of

$$
\frac{\partial u}{\partial s}=-J \frac{\partial u}{\partial t}-\nabla H(t, u(s, t)) .
$$

This equation is nothing but a Cauchy-Riemann equation perturbed by a nonlinear term of order 0 :

$$
\bar{\partial} u+\nabla H(t, u)=0
$$

where $\bar{\partial} u=\frac{\partial u}{\partial s}+J \frac{\partial u}{\partial t}$.

Proposition 2.5. If $u: \mathbb{R} \times S^{1} \rightarrow T^{2 n}$ is a solution of the perturbed CauchyRiemann equation, then we have:

$$
\begin{aligned}
\Phi_{H}\left(u_{s_{1}}\right)-\Phi_{H}\left(u_{s_{0}}\right) & =-\int_{s_{0}}^{s_{1}}\left\|\operatorname{grad} \Phi_{H}\right\|_{L^{2}}^{2} d s d t \\
& =-\int_{s_{0}}^{s_{1}} \int_{S^{1}}\left\|J \frac{\partial u}{\partial t}+\nabla H(t, u)\right\|^{2} d s d t .
\end{aligned}
$$

\section{6 $\quad L^{2}$-gradient of the action for a general symplectic man- ifold}

This requires the choice of a compatible almost complex structure.

Definition 2.6. An almost complex structure $J$ on the manifold $M$ is a linear isomorphism of the tangent space TM such that $J^{2}=-I$. If $\omega$ is a symplectic structure, $J$ is said $\omega$-compatible if $\omega(\xi, J \eta)$ is a scalar product.

Proposition 2.7. The space $\mathcal{J}(\omega)$ of the almost complex structures $\omega$-compatible is contractible (in particular non-empty).

Proof. This is based on the polar decomposition of a linear isomorphism of $T_{a} M$. Let $g$ be an auxiliary Riemannian metric on $M$. We have $\omega(\xi, \eta)=$ $g(\xi, \Omega \eta)$ and ${ }^{t} \Omega=-\Omega$ is non-singular. There exists a unique symmetric isomorphism $S$, positive definite, such that $S^{2}=\Omega^{t} \Omega$. Let us put $J=\Omega^{-1} S$. One easily checks:

- $J$ is $g$-unitary,

- $J^{-1} S J$ is symmetric positive definite and it is a square root of $\Omega^{t} \Omega$, so $J^{-1} S J=S$, 
- $S^{2} J^{-2}=\left(S J^{-1}\right)^{2}=\Omega^{2}=-S^{2}$, and then $J^{2}=-I$,

- $\omega(\xi, J \eta)=g(\xi, \Omega J \eta)=g(\xi, S \eta)$ is a scalar product.

Finally the $1-1$ correspondence $g \leftrightarrow J$ answers the question.

For the sequel we choose such a $J$ and we define the Riemannian metric

$$
<\xi, \eta>=\omega(\xi, J \eta) .
$$

If $\nabla H$ is the gradient of $H$ ( $t$ being kept fixed) with respect to this metric, the Hamiltonian system reads

$$
\dot{x}=J(x) \nabla H(t, x) .
$$

By a similar calculation to the case of $M=T^{2 n}$, the $L^{2}$-gradient of the action functional is given by

$$
\operatorname{grad} \Phi_{H}(x)=J(x) \dot{x}+\nabla H(t, x)
$$

and the equation of the gradient lines of $\Phi_{H}$ reads:

$$
\frac{\partial u}{\partial s}+J(u) \frac{\partial u}{\partial t}+\nabla H(t, u)=0
$$

\section{A brief survey of Morse theory}

\subsection{Dynamical systems point of view}

Definition 3.1. A function $H: M \rightarrow \mathbb{R}$ is called a Morse function if for any $a \in \operatorname{crit} H, d^{2} H(a): T_{a} M \times T_{a} M \rightarrow \mathbb{R}$ is non-degenerate. The Morse index $\operatorname{ind}_{a} H=\operatorname{ind}(\mathrm{a})$ is the maximal dimension of a subspace in $T_{a} M$ on which $d^{2} H(a)$ is negative definite.

As a consequence of the implicit function theorem the critical points of a Morse function are isolated.

Theorem 3.2. (Thom [54]) When $M$ is closed the set of Morse functions is open and dense in $C^{\infty}(M, \mathbb{R})$. In particular to be a Morse function is a generic property in the sense of Baire.

Lemma 3.3. (Morse's Lemma) If a is a Morse critical point of $H$ there exist local coordinates around a such that

$$
H(x)=H(a)-x_{1}^{2}-\ldots-x_{i}^{2}+x_{i+1}^{2}+\ldots
$$

where $i=\operatorname{ind}_{a} H$. 


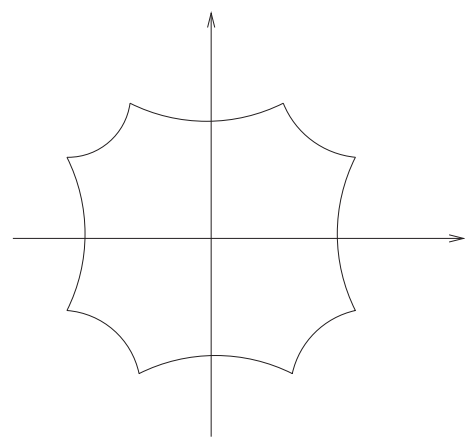

Figure 3

Such a chart is called a Morse chart. It contains Morse models an example of which is drawn in figure 3. A Morse model is a compact domain in a Morse chart whose frontier consists of three parts: the lower (resp. upper) side which is contained in a regular level set of $H$ below (resp. above) the critical value, and the lateral side which is a union of gradient segments joining both other sides. We will choose a Riemannian metric on $M$ which coincides with the Euclidean metric in some Morse charts around all the critical points. We look at the descending gradient lines, solutions of

$$
\dot{x}=-\nabla H(x) .
$$

Let $\chi_{t}$ be its flow. The unstable (resp. stable) manifold of $a$ is

$$
W^{u}(a)\left(\operatorname{resp} . W^{s}(a)\right):=\left\{x \in M \mid \chi_{t}(x) \underset{t \rightarrow \mp \infty}{\longrightarrow} a\right\} .
$$

According to Morse's Lemma and the choice of the Riemannian metric, $W^{u}(a) \cong$ $\mathbb{R}^{\text {ind }_{a} H}$.

Theorem 3.4. (Smale [52]) A generic metric (among those which coincide with a given metric near the critical points) meets the Smale transversality condition: the stable and unstable manifolds are mutually transversal.

Comment. The reason why the extra (closed) condition imposed to the metrics does not imply loss of genericity is the following. Starting from a metric $g_{0}$, in order to make the stable and unstable manifolds mutually transversal it is sufficient to modify $g_{0}$ near a family of regular level sets made of one between each pair of consecutive critical level sets. 
The next theorem does not require the Smale transversality condition. On a closed manifold it is elementary to see that, for any initial condition, the corresponding solution of the gradient equation tends to a critical point when $t \rightarrow \pm \infty$. Let $\mathcal{M}$ denote the set of solutions (or gradient lines) and, for $a, b \in$ crit $H$, let $\mathcal{M}(a, b)$ denote the set of gradient lines descending from $a$ to $b$ :

$$
\mathcal{M}=\underset{a, b \in \operatorname{crit} H}{\cup} \mathcal{M}(a, b),
$$

including $\mathcal{M}(a, a)$ which is nothing but the constant $a$. Here a gradient line means a parametrized gradient line. We have a natural evaluation map

$$
\begin{aligned}
e v: & \mathcal{M} \rightarrow M \\
& \gamma \mapsto \gamma(0)
\end{aligned}
$$

and a right action of $\mathbb{R}$ on $\mathcal{M}$ by translation of time:

$$
(\gamma \in \mathcal{M}, s \in \mathbb{R}) \mapsto(\gamma \cdot s)(t):=\gamma(s+t) .
$$

An equivalence class $\bar{\gamma}$ under this action is an unparametrized gradient line or a connecting orbit.

Theorem 3.5. If $M$ is a closed manifold and $\mathcal{M}$ is endowed with the $C_{\text {loc }}^{\infty}(\mathbb{R}, M)$ topology, then we have the following:

1) $\mathcal{M}$ is a compact set and ev $: \mathcal{M} \rightarrow M$ is a homeomorphism.

2) If $\gamma_{n} \rightarrow \gamma$ is a converging sequence in $\mathcal{M}$, with $\gamma_{n} \in \mathcal{M}(a, b)$ for all $n$, then there exist a subsequence (still denoted by $\gamma_{n}$ ) and :

- critical points $a_{0}=a, a_{1}, \ldots, a_{\ell}, a_{\ell+1}=b$,

- for each $k \in\{0, \ldots, \ell\}$, a sequence of real numbers $\left(s_{n}^{k}\right)_{n}$ and a gradient line $\gamma^{k} \in \mathcal{M}\left(a_{k}, a_{k+1}\right)$

such that $\gamma_{n} \cdot s_{n}^{k} \rightarrow \gamma^{k}$ for every $k$.

3) The limit $\gamma$ is one of the $\gamma^{k}$ 's (up to a finite shift of the variable) if $\gamma$ is not constant, or one of the $a_{k}$ 's if it is.

The $(\ell+1)$-uple of connecting orbits $\left(\bar{\gamma}^{0}, \ldots, \bar{\gamma}^{\ell}\right)$ is called a broken connecting orbit from $a$ to $b$.

Proof. 1) This is just the Cauchy-Lipschitz theorem.

2) There is a simple proof of this point using Morse models. But we take care to give a proof which also works in infinite dimension. We look at a regular value $h_{1}$ of $H$ less than $H(a)$ so that $\left[h_{1}, H(a)\right.$ [ contains no critical values. Let $s_{n}^{1}$ be real numbers such that $\left(H \circ \gamma_{n}\right)\left(s_{n}^{1}\right)=h_{1}$. Taking a subsequence if necessary, $\gamma_{n} \cdot s_{n}^{1} \rightarrow \gamma^{1}$ in $\mathcal{M}$ and $\gamma^{1}$ belongs to some $\mathcal{M}\left(a_{0}, a_{1}\right)$. We leave as an exercise to prove that $a_{0}=a$ (argue as below). We have:

$$
H\left(a_{1}\right)=\inf _{T} \lim _{n} H\left(\gamma_{n}\left(s_{n}^{1}+T\right)\right) .
$$


For the next step we take a regular value $h_{2}$ of $H$ with no critical values in $\left[h_{2}, H\left(a_{1}\right)\left[\right.\right.$. Let $s_{n}^{2}$ be real numbers such that $\left(H \circ \gamma_{n}\right)\left(s_{n}^{2}\right)=h_{2}$. Up to taking a suitable subsequence, we have:

$$
\gamma_{n} \cdot s_{n}^{2} \rightarrow \gamma^{2} \in \mathcal{M}\left(a_{1}^{\prime}, a_{2}\right) .
$$

Certainly $s_{n}^{2}-s_{n}^{1}$ is unbounded, if not $\gamma^{2}=\gamma^{1}$ up to a finite shift of the variable, and

$$
h_{2}=H\left(\gamma_{n}\left(s_{n}^{2}\right)\right)=H\left(\gamma^{2}(0)\right)>H\left(a_{1}\right),
$$

contradicting $h_{2}<H\left(a_{1}\right)$. Hence, for every $T>0$ and $n$ big enough we have

$$
H\left(\gamma_{n}\left(s_{n}^{2}-T\right)\right)<H\left(\gamma_{n}\left(s_{n}^{1}+T\right)\right)
$$

which yields $H\left(a_{1}^{\prime}\right) \leq H\left(a_{1}\right)$. Since $\left[h_{2}, H\left(a_{1}\right)\right.$ [ contains no critical values, we have $H\left(a_{1}^{\prime}\right)=H\left(a_{1}\right)$.

Let us prove that $a_{1}^{\prime}=a_{1}$. If not, there exist small disjoint open balls $B$ and $B^{\prime}$ centered at $a_{1}$ and $a_{1}^{\prime}$ whose closure contains no other critical points of $H$ other than their centers. There exists $\tau$ such that $\gamma^{1}(t) \in B$ and $\gamma^{2}(-t) \in B^{\prime}$ for every $t \geq \tau$. For $n$ big enough, $\gamma_{n}\left(s_{n}^{1}+\tau\right) \in B$ and $\gamma_{n}\left(s_{n}^{2}-\tau\right) \in B^{\prime}$. So, for every $n$, there exists $\left.\theta_{n} \in\right] s_{n}^{1}+\tau, s_{n}^{2}-\tau$ [ such that $\gamma_{n}\left(\theta_{n}\right) \in S$, where $S$ is the frontier of $B$. Taking a suitable subsequence we have $\gamma_{n} \cdot \theta_{n} \rightarrow \gamma^{\prime} \in \mathcal{M}$. The sequence $\theta_{n}-s_{n}^{1}$ is unbounded. If not, as above, $\gamma^{\prime}=\gamma^{1}$ up to a finite shift; but $\gamma^{1}(] \tau,+\infty[)$ does not meet $S$; contradiction. In the same way $s_{n}^{2}-\theta_{n}$ is unbounded and the limit values of $H \circ \gamma^{\prime}$ satisfy

$$
H\left(a_{1}\right) \geq \sup H \circ \gamma^{\prime}(\mathbb{R}) \geq \inf H \circ \gamma^{\prime}(\mathbb{R}) \geq H\left(a_{1}^{\prime}\right)=H\left(a_{1}\right) .
$$

Therefore $\gamma^{\prime}$ is constant and $\gamma^{\prime}(0) \in S$ is a critical point of $H$. Contradiction; so $a_{1}^{\prime}=a_{1}$. The other steps go in the same way with a slight modification for $a_{\ell+1}=b$.

3) Consider the case where $H(\gamma(0)) \in] H\left(a_{k+1}\right), H\left(a_{k}\right)$ [ for some $k$. Suppose $s_{n}^{k}$ is unbounded; let us say $s_{n}^{k} \rightarrow+\infty$ (the other cases are left to the reader). Hence

$$
\lim H\left(\gamma_{n}(0)\right) \geq \sup H\left(\gamma^{k}(\mathbb{R})\right)=H\left(a_{k}\right) .
$$

So $s_{n}^{k}$ is bounded and $\gamma=\gamma^{k}$ up to a finite shift of the variable.

If now $H(\gamma(0))=H\left(a_{k}\right)$, then necessarily $s_{n}^{k} \rightarrow+\infty$ ans $s_{n}^{k-1} \rightarrow-\infty$ and we conclude that $H \circ \gamma$ is constant. But $\bar{\gamma}$ is a gradient line. So it is a critical point $c$. As in the end of 2) $c \neq a_{k}$ leads to a contradiction with the fact that the critical points are isolated.

From now on, we assume the Smale transversality condition as in theorem 3.4. Unfortunately in spite of the above theorem the picture of the closure of $\mathcal{M}(a, b)$ is still a little difficult to describe, (but it is done in [33]). If we look at the quotient space $\mathcal{M} / \mathbb{R}$ of the connecting orbits, the picture is horrible because this space is not Hausdorff. But there is an abstract compactification of $\mathcal{M}(a, b) / \mathbb{R}$ 
which has a very nice structure as it is explained in the statement below, which is a direct consequence of the transversality condition and of theorem 3.5.

\section{Corollary 3.6.}

1) $\mathcal{M}(a, b) / \mathbb{R}$ is a manifold of dimension $\operatorname{ind}(a)-\operatorname{ind}(b)-1$. In particular if $\operatorname{ind}(b) \geq \operatorname{ind}(a), \mathcal{M}(a, b) / \mathbb{R}$ is empty.

2) There is a compact manifold with boundary and corners $\widehat{\mathcal{M}(a, b)} / \mathbb{R}$ formed by adding all broken orbits from a to $b$.

Part 1) is obvious since $W^{u}(a) \cap W^{s}(b)$ is a manifold whose dimension is ind $(a)$ ind $(b)$, and the action of $\mathbb{R}$ on it is free and proper. A proof of part 2) can be read in [31] or [46]. We will detail below the easy particular case when the difference of indices is 1 or 2 .

\section{Corollary 3.7.}

1) If $\operatorname{ind}(a)-\operatorname{ind}(b)=1, \mathcal{M}(a, b) / \mathbb{R}$ is made of finitely many points.

2) If $\operatorname{ind}(a)-\operatorname{ind}(b)=2, \widehat{\mathcal{M}(a, b)} / \mathbb{R}$ is made of a finite number of closed intervals or circles.

Proof. 1) Due to the indices there are no broken connecting orbits from $a$ to $b$ and we have to show $\mathcal{M}(a, b) / \mathbb{R}$ is already a compact set. Let $\left(\bar{\gamma}_{n}\right)_{n}$ be a sequence in the quotient space. It can be lifted to $\mathcal{M}(a, b)$ as a sequence $\left(\gamma_{n}\right)_{n}$ such that $\gamma_{n}(0)$ belongs to a given regular level set. Then, according to theorem 3.5 , every cluster point of this sequence in $\mathcal{M}$ belongs to $\mathcal{M}(a, b)$. So $\mathcal{M}(a, b) / \mathbb{R}$ is compact. Being a 0 -dimensional manifold it is a finite set.

2) Here a broken connecting orbit is made of exactly two pieces $\left(\bar{\gamma}_{0}, \bar{\gamma}_{1}\right)$. Let $c$ be the intermediate critical point. Let $k$ be its Morse index and $n$ the ambient dimension. We look at a Morse model about $c$. The upper side $V_{+}$, contained in $\{H=H(c)+\varepsilon\}$, is diffeomorphic to $D^{k} \times S^{n-k-1}$ and $W^{u}(a)$ traces a $k$ manifold $\Sigma_{+}$on $V_{+}$which meets $0 \times S^{n-k-1}$, the trace of $W^{s}(c)$, transversally in a single point $p$ corresponding to $\bar{\gamma}_{0}$. The lower side $V_{-}$of the Morse model, contained in $\{H=H(c)-\varepsilon\}$, is diffeomorphic to $S^{k-1} \times D^{n-k}$. The gradient lines yield a diffeomorphism

$$
G: V_{+} \backslash\left(0 \times S^{n-k-1}\right) \rightarrow V_{-} \backslash\left(S^{k-1} \times 0\right) .
$$

If we use polar coordinates, $\left.\left.\varphi \in S^{k-1}, \psi \in S^{n-k-1}, r \in\right] 0,1\right]$, a point of $V_{+}$is given by $((\varphi, r), \psi)$, a point of $V_{-}$is given by $(\varphi,(\psi, r))$ and, when $r \neq 0$,

$$
G(\varphi, r, \psi)=(\varphi, \psi, r) .
$$

With the help of this model it is easy to see that $G\left(\Sigma_{+} \backslash p\right)$ is a punctured manifold which compactifies as a manifold with boundary $\Sigma_{-}$when adding $S^{k-1} \times 0$ as a boundary. Now the trace of $W^{s}(b)$ on $V_{-}$is a $(n-k)$-dimensional manifold which intersects $S^{k-1} \times 0$ transversally in exactly one point corresponding to $\bar{\gamma}_{1}$. 
Therefore its intersection with $\Sigma_{-}$is a 1-dimensional manifold with boundary. As the interior points of this arc represent gradient lines descending directly from $a$ to $b$, this arc provides $\widehat{\mathcal{M ( a , b )}} / \mathbb{R}$ with a chart of a 1-manifold with boundary near $\left(\bar{\gamma}_{0}, \bar{\gamma}_{1}\right)$.

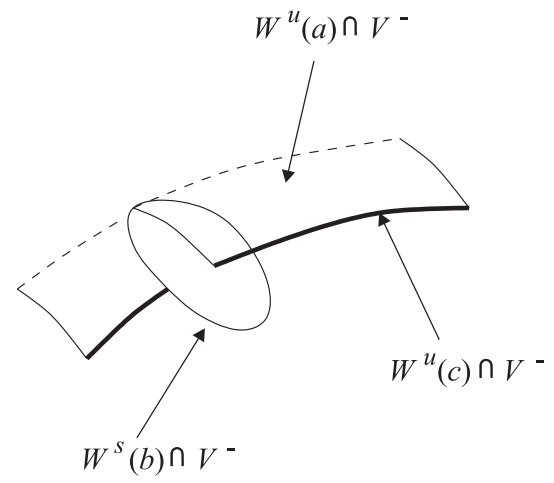

Figure 4: Traces of stable/unstable manifolds in the lower side $V_{-}$of the Morse model.

\subsection{The Morse-Smale-Witten complex}

This complex is also called the Thom-Smale complex because of the note by $\mathrm{R}$. Thom [53] which is the very beginning of this story. But the idea of transversality was missing and the right statement was given by S. Smale in [52]. More recently E. Witten rediscovered this complex by another approach.

For simplicity we do not take the orientations into account. Therefore all the coefficients are in $\mathbb{Z} / 2$. Here $H$ will be a Morse function on the closed manifold $M$ and $g$ will be a generic Riemannian metric as in theorem 3.4.

Notation 3.8. $C_{k}(H, g)$ is the $\mathbb{Z} / 2$-vector space generated by $\operatorname{crit}_{k}(H)$, the set of critical points of index $k$. For $k \notin\{0, \ldots, \operatorname{dim} M\}, C_{k}=0$.

If $a \in \operatorname{crit}_{k}(H)$ and $b \in \operatorname{crit}_{k-1}(H), n(a, b)$ denotes the number of connecting orbits from $a$ to $b$.

The boundary operator $\partial_{k}: C_{k}(H, g) \rightarrow C_{k-1}(H, g)$ is defined by

$$
\partial_{k}(a)=\sum_{b \in \mathrm{crit}_{k-1} H} n(a, b) b .
$$




\section{Theorem 3.9.}

1) $\partial_{k} \circ \partial_{k+1}=0$.

2) $H_{k}(H, g):=\frac{\operatorname{ker} \partial_{k}}{\operatorname{im} \partial_{k+1}}$ is a $\mathbb{Z} / 2$-vector space of finite dimension.

3) (Poincaré duality) $H_{k}(H, g)$ and $H_{n-k}(-H, g)$ are dual one to the other in a canonical way.

\section{Proof.}

1) If $b \in \operatorname{crit}_{k-1}(H)$ contributes to $\partial_{k} \circ \partial_{k+1}(a)$, this means there exists a broken orbit $(a, c, b)$ where $c$ is some critical point of index $k$. It corresponds to an endpoint of some interval in $\widehat{\mathcal{M ( a , b )}} / \mathbb{R}$. Then the other endpoint of this interval cancels the contribution mod 2 of $c$ to the coefficient of $b$ in $\partial_{k} \circ \partial_{k+1}(a)$.

2) This is clear because $C_{k}(H, g)$ is finitely generated.

3) If $a$ is a critical point of $H$ of index $k$, it is a critical point of $-H$ of index $n-k$. If $\gamma$ is a connecting orbit descending from $a$ to $b$ for $H$, then $\gamma^{*}$, that is $\gamma$ traversed in the opposite way, is a connecting orbit descending from $b$ to $a$ for $-H$. Let us consider the pairing

$$
C_{k}(H, g) \otimes C_{n-k}(-H, g) \rightarrow \mathbb{Z} / 2
$$

defined at the level of the critical points by $\langle a, b\rangle=1$ if and only if $a=b$. According to the definition of the boundary operator, the one from $C_{*}(H, g)$ is the transpose of the one from $C_{*}(-H, g)$.

\section{Theorem 3.10. (Continuation theorem)}

1) If $\left(H_{\lambda}, g_{\lambda}\right), \lambda \in[0,1]$, is a generic path of pairs, each being formed with a function and a Riemannian metric, it induces a morphism

$$
\Phi_{*}: H_{*}\left(H_{0}, g_{0}\right) \rightarrow H_{*}\left(H_{1}, g_{1}\right) .
$$

2) $\Phi_{*}$ does not depend on the chosen path.

3) (Naturality) If we have a diagram

$$
H_{*}\left(H_{0}, g_{0}\right) \stackrel{\Phi_{*}^{10}}{\longrightarrow} H_{*}\left(H_{1}, g_{1}\right) \stackrel{\Phi_{*}^{21}}{\longrightarrow} H_{*}\left(H_{2}, g_{2}\right) \stackrel{\Phi_{*}^{02}}{\longrightarrow} H_{*}\left(H_{0}, g_{0}\right),
$$

then $\Phi_{*}^{02} \circ \Phi_{*}^{21} \circ \Phi_{*}^{10}=\mathrm{Id}$. In particular $\Phi_{*}^{10}$ is an isomorphism whose inverse is $\Phi_{*}^{01}$.

This statement allows us to define $H_{*}(M, \mathbb{Z} / 2)$ as the "common value" of all homologies $H_{*}(H, g)$. Notice also that the functions from the path in 1) are allowed to be non-Morse for a finite number of values of the parameter. 
Proof. We only pay attention to the first part, whose proof yields easily the other parts. We will show that the given path allows us to define a morphism of complexes

$$
\Phi: C_{*}\left(H_{0}, g_{0}\right) \rightarrow C_{*}\left(H_{1}, g_{1}\right),
$$

which means a morphism commuting with the boundary operators. It is obtained in the following way according to an idea of Floer. One integrates the differential equation

$$
\frac{d x}{d \lambda}=-\nabla H_{\lambda} .
$$

For a generic path, there are finitely many connecting orbits from critical points of $H_{0}$ to critical points of $H_{1}$ whose indices are the same.

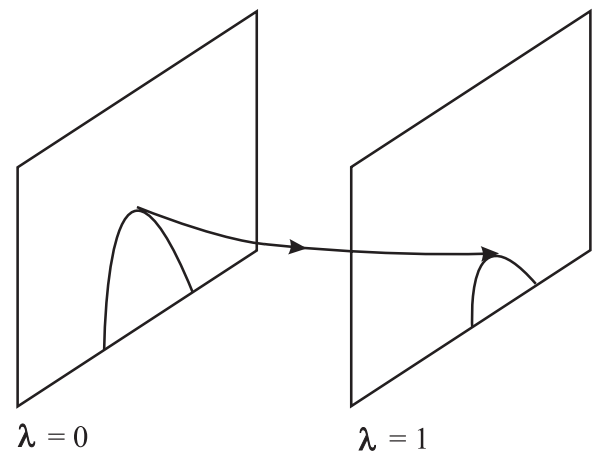

Figure 5: A gradient line of $K$ descending from $M \times\{0\}$

- A way to see this is to introduce a smooth decreasing function $\rho(\lambda)$ whose derivative vanishes exactly at the endpoints of $[0,1]$ to order 1 . Let $K$ : $M \times[0,1] \rightarrow \mathbb{R}$ be defined by $K(x, \lambda)=H_{\lambda}(x)+\rho(\lambda)$. If $\rho$ decreases strongly enough, $d K$ vanishes only at the critical points of $H_{i}$ in $M \times$ $\{i\}$ for $i=0,1$. The gradient lines on the product yields the desired connecting orbits. Observe that a critical point $a$ of $H_{0}$ satisfies $\operatorname{ind}_{a} K=$ $\operatorname{ind}_{a} H_{0}+1$. But a critical point $a$ of $H_{1}$ satisfies $\operatorname{ind}_{a} K=\operatorname{ind}_{a} H_{1}$.

Now, for $a \in \operatorname{crit}_{k}\left(H_{0}\right)$, we set

$$
\Phi(a)=\sum_{b} n(a, b) b
$$

where the sum is taken over $\operatorname{crit}_{k}\left(H_{1}\right), n(a, b)$ being the number of connecting orbits from $a$ to $b \bmod 2$. 
The fact that $\Phi$ commutes with $\partial$ can be deduced from $\partial \circ \partial=0$ when we look at the above mentioned function $K$ on the product $M \times[0,1]$.

\section{Compactness}

\subsection{Finite energy}

We recall the situation: $(M, \omega)$ is a closed symplectic manifold, $H: S^{1} \times M \rightarrow$ $\mathbb{R}$ is a Hamiltonian 1-periodic in time, $J$ is an almost complex structure $\omega$ compatible and $\langle\xi, \eta\rangle=\omega(\xi, J \eta)$ is the associated Riemannian metric. We have an action functional $\Phi_{H}$ defined on the space $\Lambda$ of contractible smooth loops in $M$ :

$$
\Phi_{H}=-\int_{D^{2}} v^{*} \omega+\int_{S^{1}} H(t, x) d t,
$$

where $x: S^{1} \rightarrow M, v: D^{2} \rightarrow M$ and $v \mid \partial D^{2}=x$. It is well defined under the homotopic assumption

$$
<\omega, \pi_{2}(M)>=0 .
$$

The critical points of $\Phi_{H}$ are the 1-periodic solutions of the Hamilton differential equation

$$
\dot{x}=J(x) \nabla H(t, x) \text {. }
$$

The descending $L^{2}$-gradient lines of $\Phi_{H}$ are the solutions of the perturbed Cauchy-Riemann equation

$$
\text { (*) } \frac{\partial u}{\partial s}+J(u) \frac{\partial u}{\partial t}+\nabla H(t, u)=0 .
$$

We define the energy of a gradient line $u$ by

$$
\begin{aligned}
E(u) & :=-\int_{-\infty}^{+\infty} \frac{d}{d s} \Phi_{H}(u(s)) d s \\
& =\frac{1}{2} \int_{\mathbb{R} \times S^{1}}\left(\left\|\frac{\partial u}{\partial s}\right\|^{2}+\left\|J(u) \frac{\partial u}{\partial t}+\nabla H(t, u)\right\|^{2}\right) d s d t .
\end{aligned}
$$

Here $u(s)$ means $u \mid\{s\} \times S^{1}$. Of course both terms from the last integrand are equal. We introduce the set of gradient lines whose energy is finite:

$\mathcal{M}=\left\{u: \mathbb{R} \times S^{1} \rightarrow M \mid\right.$ smooth, contractible, solution of $\left.(*), E(u)<\infty\right\}$.

\section{Proposition 4.1.}

1) If $u \in \mathcal{M}, \Phi_{H}(u(s))$ tends to a critical value $\Phi\left(u_{ \pm}\right)$when $s$ goes to $\pm \infty$, where $u_{ \pm}$is a 1-periodic solution of the Hamilton equation and there is at least one accumulating point of $\{u(s) \mid s \rightarrow \pm \infty\}$ in $\Lambda$.

2) There exists a constant $C>0$ such that $\Phi_{H}(\mathcal{M}) \subset[-C, C]$.

3) For $u \in \mathcal{M}, E(u) \leq 2 C$. 
Proof.

1) For $u \in \mathcal{M}$ we have:

$$
\int_{-\infty}^{+\infty} \int_{S^{1}}\|\dot{u}-J(u) \nabla H(t, u)\|^{2} d s d t<+\infty
$$

where $\dot{u}=\frac{\partial u}{\partial t}$. Then there exists a sequence $s_{k} \rightarrow+\infty$ (or $\left.-\infty\right)$ such that

$$
(* *) \quad\left\|\dot{u}_{k}-J\left(u_{k}\right) \nabla H\left(t, u_{k}\right)\right\|_{L^{2}} \rightarrow 0
$$

where $u_{k}:=u\left(s_{k}\right)$. In order to speak of $L^{2}$ we use an embedding of $M$ into some $\mathbb{R}^{N}$.

As $\nabla H$ is bounded (compactness of $M$ ), there exists $B>0$ such that, for every $k,\left\|\dot{u}_{k}\right\|_{L^{2}} \leq B$. This bound implies that the family of the $u_{k}$ 's is equicontinuous. Indeed:

$$
u_{k}\left(t_{1}\right)-u_{k}\left(t_{0}\right)=\int_{t_{0}}^{t_{1}} \dot{u}_{k}=\int_{S^{1}} \mathbf{1}_{\left[t_{0}, t_{1}\right]} \dot{u}_{k} \leq\left[t_{1}-t_{0}\right]^{1 / 2}\left\|u_{k}\right\|_{L^{2}} \leq B\left[t_{1}-t_{0}\right]^{1 / 2} .
$$

Thus the family has a compact closure in $C^{0}\left(S^{1}, M\right)$ (Arzelà-Ascoli Theorem). Taking a subsequence if necessary $u_{k} \rightarrow u_{+}$(or $\left.u_{-}\right)$in $C^{0}$. Due to $(* *), \dot{u}_{k}$ is a Cauchy sequence in $L^{2}$ converging to the continuous function $v=J\left(u_{+}\right) \nabla H\left(t, u_{+}\right)$. By the dominated convergence theorem,

$$
u_{+}(t)-u_{+}(0)=\int_{0}^{t} v(\theta) d \theta
$$

Therefore $u_{+}$is $C^{1}$ and a solution of the Hamilton equation. Then it is $C^{\infty}$. Of course $\Phi_{H}\left(u_{k}\right) \rightarrow \Phi_{H}\left(u_{+}\right)$with $k \rightarrow \infty$. But, as $\Phi_{H}(u(s))$ is monotone, we get

$$
\Phi_{H}(u(s)) \underset{s \rightarrow+\infty}{\longrightarrow} \Phi_{H}\left(u_{+}\right) .
$$

2) The Arzelà-Ascoli Theorem implies easily that the set of the 1-periodic solutions of the Hamilton equation is compact in $C^{\infty}\left(S^{1}, M\right)$. Then the action functional is bounded on this set.

3) This is clear because $E(u)=\Phi_{H}\left(u_{-}\right)-\Phi_{H}\left(u_{+}\right)$.

Corollary 4.2. (Exercise, [29] p. 236) $\mathcal{M}$ is closed in $C_{\text {loc }}^{\infty}\left(\mathbb{R} \times S^{1}, M\right)$.

Remark 4.3. As pointed out by David Hermann, the arguments used in the above proposition imply that the action functional meets the so-called PalaisSmale condition: if $\left(x_{n}\right)_{n}$ is a sequence in $\Lambda$ such that $\operatorname{grad} \Phi\left(x_{n}\right)$ tends to 0 in $L^{2}$, then there is a subsequence converging to a critical point of $\operatorname{grad} \Phi$. But according to remark 2.4, this observation is of no help for the compactness of $\mathcal{M}$. 


\subsection{Compactness theorem}

Theorem 4.4. Under the assumption $\left\langle\omega, \pi_{2}(M)\right\rangle=0$ (which allows us to define the action functional) we have the following:

1) There exists $A>0$ such that, for every $u \in \mathcal{M}$ and $(s, t) \in \mathbb{R} \times S^{1}$, $\|\nabla u(s, t)\| \leq A$.

2) $\mathcal{M}$ is compact in $C_{\text {loc }}^{\infty}\left(\mathbb{R} \times S^{1}, M\right)$.

Proof. $1 \Longrightarrow 2$

By 1) and the Arzelà-Ascoli theorem, $\mathcal{M}$ has a compact closure in $C_{\text {loc }}^{0}(\mathbb{R} \times$ $\left.S^{1}, M\right)$. Moreover the Cauchy-Riemann equation is elliptic. Then the so-called elliptic regularity implies that the solutions are $C^{\infty}$, any $C^{0}$-limit of solutions is a solution and both $C^{0}$ and $C^{\infty}$ topologies coincide on $\mathcal{M}$. Hence $\mathcal{M}$ is $C^{0}$-compact and then $C^{\infty}$-compact.

Proof of 1). Here it is more convenient to think of $u \in \mathcal{M}$ as defined on $\mathbb{R}^{2}$ instead of $\mathbb{R} \times S^{1}$, in particular when a rescaling is performed. If 1 ) is not true, there exist sequences $u_{k} \in \mathcal{M}$ and $\left(s_{k}, t_{k}\right) \in \mathbb{R}^{2}$ such that $\left\|\nabla u_{k}\left(s_{k}, t_{k}\right)\right\| \rightarrow \infty$. Let $\varepsilon_{k}>0$ such that $\varepsilon_{k} \rightarrow 0$ and $\varepsilon_{k}\left\|\nabla u_{k}\left(s_{k}, t_{k}\right)\right\| \rightarrow \infty$.

Lemma 4.5. (Ekeland [18]) Let $g: X \rightarrow \mathbb{R}_{+}$be a continuous function on a complete metric space. Given $x_{0} \in X$ and $\varepsilon_{0}>0$, there exist $x^{*} \in X$ and $0<\varepsilon^{*} \leq \varepsilon_{0}$ such that:

1) $d\left(x^{*}, x_{0}\right) \leq 2 \varepsilon_{0}$,

2) $\varepsilon^{*} g\left(x^{*}\right) \geq \varepsilon_{0} g\left(x_{0}\right)$,

3) $g(x) \leq 2 g\left(x^{*}\right)$ for every $x \in B\left(x^{*}, \varepsilon^{*}\right)$.

Proof. If $\left(x_{0}, \varepsilon_{0}\right)$ satisfies $g(x) \leq 2 g\left(x_{0}\right)$ for every $x \in B\left(x_{0}, \varepsilon_{0}\right)$ one takes $x^{*}=x_{0}$ and $\varepsilon^{*}=\varepsilon_{0}$. If not we choose $x_{1} \in B\left(x_{0}, \varepsilon_{0}\right)$ with $g\left(x_{1}\right)>2 g\left(x_{0}\right)$ and $\varepsilon_{1}=\varepsilon_{0} / 2$ and so on. The complete proof is left to the reader.

Let us apply this lemma to $g=\left\|\nabla u_{k}(s, t)\right\|$. Then there exist other $\varepsilon_{k}$ and $\left(s_{k}, t_{k}\right)$ such that:

- $\varepsilon_{k}\left\|\nabla u_{k}\left(s_{k}, t_{k}\right)\right\| \rightarrow \infty$ with $k$,

- $2\left\|\nabla u_{k}\left(s_{k}, t_{k}\right)\right\| \geq\left\|\nabla u_{k}(s, t)\right\|$ for every $(s, t) \in B\left(\left(s_{k}, t_{k}\right), \varepsilon_{k}\right)$.

The sequel of the proof consists in some rescaling:

$$
v_{k}=u_{k}\left(\left(s_{k}, t_{k}\right)+\frac{(s, t)}{R_{k}}\right)
$$

where $R_{k}=\left\|\nabla u_{k}\left(s_{k}, t_{k}\right)\right\|$. The formula makes sense when $u_{k}$ is thought of as defined on $\mathbb{R}^{2}$, as we said above. We have some obvious properties:

- $\left\|\nabla v_{k}(0,0)\right\|=1$, 
- $\left\|\nabla v_{k}(s, t)\right\| \leq 2$ on the "big" disk $D_{k}=B\left(\underline{0}, \varepsilon_{k} R_{k}\right)$ centered at the origin,

- $\frac{\partial v_{k}}{\partial s}+J\left(v_{k}\right) \frac{\partial v_{k}}{\partial t}+\frac{1}{R_{k}} \nabla H\left(t_{k}+\frac{t}{R_{k}}, v_{k}\right)=0$.

Lemma 4.6. For $k$ big enough, we have

$$
\int_{D_{k}}\left\|\frac{\partial v_{k}}{\partial s}\right\|^{2}+\left\|\frac{\partial v_{k}}{\partial t}\right\|^{2} \leq 7 C .
$$

Proof. Let $B_{k}:=B\left(\left(s_{k}, t_{k}\right), \varepsilon_{k}\right)$. It is a small disk in $\mathbb{R}^{2}$ or in the cylinder as well. The first identity below is nothing but the change of variables in integrals and the rest is obvious.

$$
\begin{aligned}
& \int_{D_{k}}\left\|\nabla v_{k}\right\|^{2}=\int_{B_{k}}\left\|\nabla u_{k}\right\|^{2} \\
& \leq \int_{B_{k}}\left\|\frac{\partial u_{k}}{\partial s}\right\|^{2}+\left\|\frac{\partial u_{k}}{\partial t}-X_{H}\left(t, u_{k}\right)+X_{H}\left(t, u_{k}\right)\right\|^{2} \\
& \leq \int_{B_{k}}\left[\left\|\frac{\partial u_{k}}{\partial s}\right\|^{2}+\left\|\frac{\partial u_{k}}{\partial t}-X_{H}\right\|^{2}\right]+\left\|X_{H}\right\|^{2}+2\left\|\frac{\partial u_{k}}{\partial t}-X_{H}\right\| \cdot\left\|X_{H}\right\| \\
& \leq \int_{B_{k}}\left[\left\|\frac{\partial u_{k}}{\partial s}\right\|^{2}+2\left\|\frac{\partial u_{k}}{\partial t}-X_{H}\right\|^{2}\right]+2\left\|X_{H}\right\|^{2} \quad\left(2 a b \leq a^{2}+b^{2}\right) \\
& \leq 3 E\left(u_{k}\right)+2 \int_{B_{k}}\left\|X_{H}\right\|^{2} \leq 6 C+2 \int_{B_{k}}\left\|X_{H}\right\|^{2} .
\end{aligned}
$$

The last inequality follows from proposition 4.1 and the last integral converges to 0 with $k \rightarrow \infty$.

Lemma 4.7. (The bubbling lemma.) Taking a subsequence if necessary, $v_{k} \rightarrow v$ in $C_{l o c}^{\infty}\left(\mathbb{R}^{2}, M\right)$ with the following properties:

- $\|\nabla v(0,0)\|=1$ (in particular $v$ is not constant),

- $\|\nabla v(s, t)\| \leq 2$ for every $(s, t) \in \mathbb{R}^{2}$.

- $\frac{\partial v}{\partial s}+J(v) \frac{\partial v}{\partial t}=0$ which means that $v$ is J-holomorphic, 
- $\int_{\mathbb{R}^{2}}\|\nabla v\|^{2}<\infty$.

Proof. Using the previous lemma, the bubbling lemma follows from usual Sobolev techniques as for $1 \Rightarrow 2$.

In order to finish the proof of theorem 4.4 let us show how the bubbling lemma leads to a contradiction. We have

$$
\int_{\mathbb{R}^{2}} v^{*} \omega=\int_{\mathbb{R}^{2}} \omega\left(\frac{\partial v}{\partial s}, \frac{\partial v}{\partial t}\right) d s d t=\int \omega\left(\frac{\partial v}{\partial s}, J(v) \frac{\partial v}{\partial s}\right)=\int\left\|\frac{\partial v}{\partial s}\right\|^{2}<\infty .
$$

Since $\frac{\partial v}{\partial s}$ and $\frac{\partial v}{\partial t}$ are isometric and orthogonal, the last integral is the area of $v$. Let us put $A(r):=\int_{B(r)} v^{*} \omega$, the area of $v(B(r))$, and $\ell(r)$ the length of $v(\partial B(r))$. We know that $A(r)$ is a bounded primitive of $\ell(r)$. Then there exists a sequence $r_{k} \rightarrow \infty$ such that $\ell\left(r_{k}\right) \rightarrow 0$ (we see the bubble).

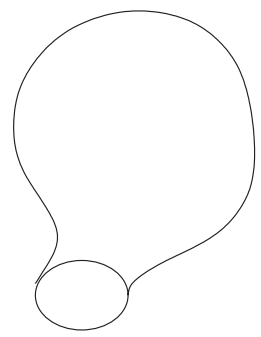

Figure 6: $J$-holomorphic disk with a small boundary.

For $k$ large, $v\left(\partial B\left(r_{k}\right)\right)$ has a small diameter and lies entirely in one of the charts from a finite atlas of $M$. Taking a subsequence if necessary all the $v\left(\partial B\left(r_{k}\right)\right)$ 's lie in the same chart $U$ where $\omega$ is exact, $\omega=d \lambda$. We have

$$
\left|\int_{v\left(\partial B\left(r_{k}\right)\right)} \lambda\right| \leq \text { const. } \ell\left(r_{k}\right)
$$

which converges to 0 with $k \rightarrow \infty$. There is a disk $\Delta_{k}$ in $U$ (maybe with singularities) whose boundary is $v\left(\partial B\left(r_{k}\right)\right)$ and Stokes formula applies and yields

$$
\int_{\Delta_{k}} \omega \rightarrow 0
$$


We remember the homotopic assumption $\left\langle\omega, \pi_{2}(M)\right\rangle=0$. So we have

$$
\int_{v\left(B\left(r_{k}\right)\right)} \omega=\left|\int_{\Delta_{k}} \omega\right| .
$$

The right-hand side converges to 0 . But the left-hand side converges to the area of $v$ which is positive because $v$ is a non constant $J$-holomorphic curve. This finishes the proof of the compactness theorem.

\subsection{Non-degeneracy condition}

In the sequel we shall assume the following generic condition: Let $\varphi_{1}$ be the time 1 map of the Hamiltonian flow; for every fixed point a of $\varphi_{1}, 1$ is not an eigenvalue of $D \varphi_{1}(a)$. By the implicit function theorem $a$ is an isolated fixed point of $\varphi_{1}$. In that case we say that the 1-periodic solutions of the Hamilton equation (or of $X_{H_{t}}$ ) are non-degenerate.

Be careful! The non-degeneracy condition is never satisfied for a Hamiltonian independent of time if there are non-constant 1-periodic orbits.

Looking at $u \in \mathcal{M}$ as a curve in $\Lambda$, the space of smooth contractible loops in $M$, we are interested in its cluster points. For this purpose it is useful to introduce the evaluation map

$$
\begin{aligned}
e v: & \mathcal{M} \rightarrow \Lambda \\
& u \mapsto u(0)
\end{aligned}
$$

(where $u(s)$ stands for $t \in S^{1} \mapsto u(s, t) \in M$ ) and the right action of $\mathbb{R}$ defined by

$$
(u \cdot \sigma)(s, t)=u(s+\sigma, t) .
$$

One checks easily that $u \in \mathcal{M}$ implies $u \cdot \sigma \in \mathcal{M}$.

Proposition 4.8. For $u \in \mathcal{M}$, any cluster point of $u(s)$ in $\Lambda$ when $s \rightarrow \pm \infty$ is a critical point of the action functional.

Proof. This is a little trickier than in finite dimension because we do not have a flow on $e v(\mathcal{M})$ (no Cauchy-Lipschitz theorem). Let $s_{k} \rightarrow+\infty$ be a sequence such that $u\left(s_{k}\right) \rightarrow \lambda \in \Lambda$. Certainly $\lambda$ belongs to $e v(\mathcal{M})$. Indeed, since $\mathcal{M}$ is compact and taking a subsequence if necessary, $u \cdot s_{k} \rightarrow v \in \mathcal{M}$ and $v(0)=\lambda$. The convergence being uniform on each compact interval $[-T,+T]$ and according to proposition 4.11 ), we deduce :

$$
\begin{aligned}
& \sup \left(\Phi_{H} \circ v\right)(\mathbb{R})=\sup _{T \rightarrow+\infty} \lim _{k} \Phi_{H}\left(u\left(s_{k}-T\right)\right)=\lim _{s \rightarrow+\infty} \Phi_{H}(u(s)), \\
& \inf \left(\Phi_{H} \circ v\right)(\mathbb{R})=\inf _{T \rightarrow+\infty} \lim _{k} \Phi_{H}\left(u\left(s_{k}+T\right)\right)=\lim _{s \rightarrow+\infty} \Phi_{H}(u(s)) .
\end{aligned}
$$

Then $\left(\Phi_{H} \circ v\right)(\mathbb{R})$ contains a single point $\Phi_{H}(\lambda)$. Since $v$ is a gradient line, this implies that $\lambda$ is a critical point. 


\section{Corollary 4.9.}

1) If all 1-periodic solutions of $X_{H_{t}}$ are non-degenerate, then for any $u \in \mathcal{M}$, $u(s) \rightarrow u_{ \pm}$in $\Lambda$ when $s \rightarrow \pm \infty$.

2) $\frac{\partial u}{\partial s}(s, t)$ tends to 0 when $s \rightarrow \pm \infty$ uniformly in $t \in S^{1}$.

Proof.

1) We consider only $s \rightarrow+\infty$. We already know that there are cluster points (propostion 4.1). The existence of a limit reduces to the uniqueness of cluster points when $s \rightarrow+\infty$. This set of cluster points is

$$
A=\bigcap_{n}(\overline{\{u(s) \mid s \geq n\}})
$$

and, as $e v(\mathcal{M})$ is compact, it is a decreasing intersection of compact connected sets. Therefore it is connected. But, by the previous proposition, it is included in the finite set $\operatorname{crit}\left(\Phi_{H}\right)$. So $A$ contains only one point.

2) For $u \in \mathcal{M}$ we have:

$$
-\frac{\partial u}{\partial s}(s, t)=\left[J(u) \frac{\partial u}{\partial t}+\nabla H(t, u)\right](s, t)
$$

and, according to 1 ), it tends to

$$
\left[J\left(x_{ \pm}\right) \dot{x}_{ \pm}+\nabla H\left(t, x_{ \pm}\right)\right](t)
$$

uniformly in $t$. But this limit is 0 since $x_{ \pm}$is a 1 -periodic solution of the Hamilton equation.

In other words any $u \in \mathcal{M}$ belongs to some $\mathcal{M}\left(x_{-}, x_{+}\right)$, the set of gradient lines of $\Phi_{H}$ descending from $x_{-}$to $x_{+}$. The right action of $\mathbb{R}$ keeps $\mathcal{M}\left(x_{-}, x_{+}\right)$ invariant and an equivalence class $\bar{u}$ is called a connecting orbit. Now it is possible to state and prove an analogue of theorem 3.52 ).

Theorem 4.10. (Broken connecting orbit theorem).

Let $u_{n} \rightarrow u$ be a converging sequence in $\mathcal{M}$, with $u_{n} \in \mathcal{M}\left(x_{-}, x_{+}\right)$for all $n$. Then there exist a subsequence (still denoted by $u_{n}$ ) and:

- 1-periodic solutions of $X_{H_{t}}, x_{0}=x_{-}, x_{1}, \ldots, x_{\ell}, x_{\ell+1}=x_{+}$

- for each $k \in\{0, \ldots, \ell\}$, a sequence of real numbers $\left(s_{n}^{k}\right)_{n}$ and a $L^{2}$ gradient line $u^{k} \in \mathcal{M}\left(x_{k}, x_{k+1}\right)$

such that $u_{n} \cdot s_{n}^{k} \rightarrow u^{k}$ when $n \rightarrow \infty$.

The $(\ell+1)$-uple of connecting orbits $\left(\bar{u}^{0}, \ldots, \bar{u}^{\ell}\right)$ is called a broken connecting orbit. The proof goes as in theorem 3.5 where the Morse function is replaced by the action functional. Intentionally we avoided making use of Morse models. We only used that the set of gradient lines is compact and the finiteness of the critical set, which is true in our infinite dimensional setting. 


\section{$5 \quad$ Floer Homology}

Here is an "easy" case of Floer's theorem we are aiming at.

Theorem 5.1. Let $(M, \omega)$ be a closed symplectic manifold. Let $H: \mathbb{R} \times M \rightarrow \mathbb{R}$ be a Hamiltonian 1-periodic in time and $X_{H}$ be its associated vector field. We assume the following:

1) $<[\omega], \pi_{2}(M)>=0$,

2) $<c_{1}(M), \pi_{2}(M)>=0$,

3) the 1-periodic solutions of $X_{H_{t}}$ are non-degenerate.

Then the number of 1-periodic solutions of the Hamilton equation is at least $\operatorname{dim}_{\mathbb{Z} / 2} H_{*}(M, \mathbb{Z} / 2)$.

Condition 2) means that the tangent fiber space, equipped with any complex structure $J$ which is $\omega$-compatible, is trivial as a complex fiber bundle over any 2 -sphere in $M$ and this condition does not depend on the chosen $J$. Actually there are more precise conclusions like Morse inequalities. But they require defining an index for each 1-periodic solution of the Hamilton equation. One could think of 1-periodic solutions as critical points of the action functional and look at its Morse index. Unfortunately this does not work. When $M=\mathbb{R}^{2 n}$, an easy calculation by Fourier transform shows that both the index and co-index are infinite. The right index to consider is the Conley-Zehnder index.

\subsection{The Conley-Zehnder index (case of $\mathbb{R}^{2 n}$ )}

Let $\dot{x}=J \nabla H(t, x)$ be the Hamiltonian differential equation under consideration and let $(x(t))$ be a non-degenerate 1-periodic solution. The linearized differential equation along $(x(t))$ reads

$$
\dot{\xi}=J S(t) \xi
$$

where $S(t):=D^{2} H(t, x(t))$, a symmetric matrix 1-periodic in $t$. Let $\left(R_{t}\right)$ be its linear flow. It is a path in $\operatorname{Sp}(2 n)$, the $2 n \times 2 n$ symplectic matrices, such that $R_{0}=I$ and $\dot{R}_{t}=J S(t) R_{t}$. The non-degeneracy condition of the 1-periodic $(x(t))$ reads :

$$
R_{1} \in \operatorname{Sp}(2 n)^{*}:=\{A \in \operatorname{Sp}(2 n) \mid \operatorname{det}(I-A) \neq 0\} .
$$

The Maslov cycle is

$$
\Sigma:=\{A \in \operatorname{Sp}(2 n) \mid \operatorname{det}(I-A)=0\} .
$$

It is a hypersurface whose singularities have codimension $\geq 3$ in $\operatorname{Sp}(2 n)$ (compare [2]). 


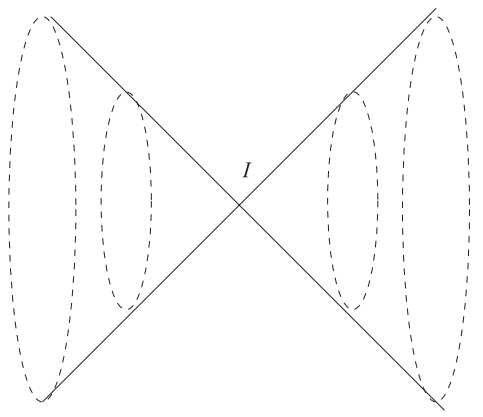

Figure 7: The Maslov cycle in $\operatorname{Sp}(2)$ near $I$.

Proposition 5.2. [15][45]

1) $\operatorname{Sp}(2 n)^{*}$ has two connected components:

$$
\operatorname{Sp}(2 n)^{ \pm}=\left\{A \mid \operatorname{det}(I-A) \in \mathbb{R}^{ \pm}\right\} .
$$

2) Any loop in $\operatorname{Sp}(2 n)^{*}$ is contractible in $\operatorname{Sp}(2 n)$.

Another proof is given in the appendix.

Theorem 5.3. (Conley-Zehnder) [15][45]

1) Given a path $\left(R_{t}\right)$ in $\mathrm{Sp}(2 n)$ from $I$ to $R_{1} \in \mathrm{Sp}(2 n)^{*}$, there exists a "natural" index $\mu\left(\left(R_{t}\right)\right) \in \mathbb{Z}$ which is invariant under homotopy as long as $R_{1}$ remains in $\operatorname{Sp}(2 n)^{*}$. Moreover it classifies the homotopy classes of such paths $\left(R_{t}\right)$.

2) We have the following sign formula

$$
\operatorname{sign} \operatorname{det}\left(I-R_{1}\right)=(-1)^{n-\mu\left(\left(R_{t}\right)\right)} .
$$

3) When $S$ is symmetric, independent of $t$ with $\|S\|<2 \pi$, then

$$
\mu((\exp t J S))=-\operatorname{ind}(S)+n,
$$

where ind $(S)$ denotes the number of negative eigenvalues of $S$.

This index is "natural" in the sense that it is invariant under conjugacy and additive with respect to direct sums. In [6] it is related to a symplectic translation number. 


\section{Sketch of proof.}

1) The polar decomposition yields a homeomorphism

$$
\operatorname{Sp}(2 n) \rightarrow Q \times \mathrm{U}(n)
$$

where $\mathrm{U}(n)$ is the unitary group, the intersection $\operatorname{Sp}(2 n) \cap \mathrm{O}(2 n)$, and where $Q$ is the subgroup of symmetric, positive definite, symplectic matrices. The space $Q$ is contractible and

$$
\operatorname{det}_{\mathbb{C}}: \mathrm{U}(n) \rightarrow S^{1}
$$

induces an isomorphism on $\pi_{1}$. Here is a hint for the contratibility of $Q$ : for $A \in Q$, let $E_{\lambda}$ be the eigenspace of eigenvalue $\lambda \geq 1$ and set $F_{\lambda}:=E_{\lambda} \oplus E_{\lambda^{-1}}$; $F_{\lambda}$ is a symplectic $J$-invariant subspace; then $A$ reads in an unique way $A=e^{J S}$ where $S$ is symmetric and anticommutes with $J$. Thus a contraction is provided by $(A, t) \mapsto e^{t J S}, t \in[0,1]$.

From the polar decomposition we get a continuous map

$$
\rho: \operatorname{Sp}(2 n) \rightarrow S^{1},
$$

which is the composition of the projection $\mathrm{Sp}(2 n) \rightarrow \mathrm{U}(n)$ followed by $\operatorname{det}_{\mathbb{C}}$. It induces an isomorphism on $\pi_{1}$. In both connected components $\operatorname{Sp}(2 n)^{ \pm}$we choose base points:

$$
\begin{aligned}
& W_{+}:=\operatorname{diag}(-1, \ldots,-1) \in \operatorname{Sp}(2 n)^{+} \\
& W_{-}:=\operatorname{diag}\left(2,-1, \ldots, \frac{1}{2},-1, \ldots\right) \in \operatorname{Sp}(2 n)^{-} .
\end{aligned}
$$

We have $\rho\left(W_{+}\right)=(-1)^{n}$ and $\rho\left(W_{-}\right)=(-1)^{n-1}$. Given a path $\left(R_{t}\right)$, one connects $R_{1}$ to $W_{ \pm}$by a path $\alpha \in \operatorname{Sp}(2 n)^{*}$ yielding a new path $\left(R_{t}^{\prime}\right)$ from $I$ to $W_{ \pm}$in the same homotopy class. In lifting $t \mapsto \rho\left(R_{t}^{\prime}\right)$ to the universal cover of $S^{1}$ we get $\rho(t)=e^{i a(t)}$ with $a(0)=0$ and $a(1) \in \pi \mathbb{Z}$. We now can define:

$$
\mu\left(\left(R_{t}\right)\right)=\frac{a(1)}{\pi} .
$$

This integer does not depend on the choice of $\alpha$ because a change of $\alpha$ has the effect of composing $\left(R_{t}^{\prime}\right)$ with a loop $\lambda$ in $\operatorname{Sp}(2 n)^{*}$ and, due to proposition 5.2 $2)$, it does not change $\mu\left(\left(R_{t}\right)\right)$. Therefore this integer is an invariant of the homotopy class of $\left(R_{t}\right)$ as long as $R_{1}$ remains in $\operatorname{Sp}(2 n)^{*}$. The fact that it is a complete invariant of the homotopy class is a direct consequence of $\rho$ being a $\pi_{1}$-isomorphism. Therefore we have the following invariance by conjugation

$$
\mu\left(\left(P_{t} R_{t} P_{t}^{-1}\right)\right)=\mu\left(\left(R_{t}\right)\right)
$$

where $t \mapsto P_{t}$ is any path in $\operatorname{Sp}(2 n)$.

2) For the sign formula we can take $R_{1}=W_{ \pm}$and any path $\alpha$ from $I$ to $W_{ \pm}$. Indeed composing $\alpha$ by a loop based at $W_{ \pm}$changes $\mu\left(\left(R_{t}\right)\right)$ by an even integer. Then calculating the invariant associated with a simple path from $I$ to 
$W_{ \pm}$yields the sign formula.

3) We only check this point on examples. For instance when $n=1$ and $S=$ $\left(\begin{array}{ll}\varepsilon & 0 \\ 0 & \varepsilon\end{array}\right)$, we have $\exp J S=\left(\begin{array}{cc}\cos \varepsilon & -\sin \varepsilon \\ \sin \varepsilon & \cos \varepsilon\end{array}\right)$ and $\rho(\exp J S)=e^{i \varepsilon}$. If $2 \pi>\varepsilon>0$, then, with the above notation, $R_{1}^{\prime}=W_{+}$and $a(1)=+\pi$. If $-2 \pi<\varepsilon<0$, then $a(1)=-\pi$. In both cases we have

$$
\mu((\exp t J S))=-\operatorname{ind}(S)+n .
$$

When $S=\left(\begin{array}{cc}-\varepsilon & 0 \\ 0 & +\varepsilon\end{array}\right), J S$ has two real eigenspaces and $\mu((\exp t J S))=0$. The index formula is still true in that case.

Remark 5.4. If $\left(U_{t}\right)$ is a loop in $\mathrm{U}(n)$ based at $I$ with $\operatorname{det}\left(U_{t}\right)=e^{i a(t)}$ and $a(0)=0$, then $a(1) / \pi$ is the usual Maslov index of this loop and it belongs to $2 \mathbb{Z}$.

Definition 5.5. In the same setting as above the Conley-Zehnder index is defined by

$$
i_{C Z}\left(\left(R_{t}\right)\right)=-\mu\left(\left(R_{t}\right)\right) .
$$

The $\mu$-index is closely related to the Maslov index while the $i_{\mathrm{CZ}}$-index is closer to the Morse index.

\subsection{Relation with a Fredholm index}

Let us consider

$$
F:=\frac{\partial}{\partial s}+J \frac{\partial}{\partial t}+S(s, t)
$$

where $S: \mathbb{R} \times S^{1} \rightarrow \operatorname{End}\left(\mathbb{R}^{2 n}\right)$ is continuous. When $s \rightarrow \pm \infty, S(s, t)$ is assumed to tend to a symmetric matrix $S_{ \pm}(t)$. Let $R_{t}^{ \pm}$be the solution of $\dot{R}=J S_{ \pm}(t) \cdot R$ with initial condition $R_{0}^{ \pm}=I$.

Theorem 5.6. If $\operatorname{det}\left(I-R_{1}^{ \pm}\right) \neq 0$, then $F: W^{1,2}\left(\mathbb{R} \times S^{1}, \mathbb{R}^{2 n}\right) \rightarrow L^{2}(\mathbb{R} \times$ $\left.S^{1}, \mathbb{R}^{2 n}\right)$ is a Fredholm operator. Its Fredholm index is given by

$$
\begin{aligned}
\operatorname{ind}(F) & =\mu\left(\left(R_{t}^{+}\right)\right)-\mu\left(\left(R_{t}^{-}\right)\right) \\
& =i_{C Z}\left(\left(R_{t}^{-}\right)\right)-i_{C Z}\left(\left(R_{t}^{+}\right)\right) .
\end{aligned}
$$

We recall that $W^{1,2}$ is the Sobolev space of functions in $L^{2}$ whose weak derivatives are in $L^{2}$. It is a Hilbert space for a suitable scalar product. The operator 
$F$ is Fredholm if it has a closed range and if its kernel and cokernel have a finite dimension. By definition its Fredholm index is

$$
\operatorname{ind}(F)=\operatorname{dim} \operatorname{ker} F-\operatorname{dim} \operatorname{coker} F .
$$

Proof. The Fredholm property and the index do not change by adding a compact operator, for instance in modifying $S$ among the bounded matrix functions. The right-hand side of the index formula does not change as long as $R_{1}^{ \pm}$remains in $\operatorname{Sp}^{*}(2 n)$. In this way we are reduced to the case when $S(s, t)$ is symmetric and independent of $t$. For every $s \in \mathbb{R}$,

$$
A(s):=J \frac{\partial}{\partial t}+S(s): W^{1,2}\left(S^{1}, \mathbb{R}^{2 n}\right) \rightarrow L^{2}\left(S^{1}, \mathbb{R}^{2 n}\right)
$$

is self-adjoint. Its spectrum is discrete. It contains 0 if and only if there exists some non-trivial 1-periodic solution of

$$
\frac{\partial \xi}{\partial t}=J S(s) \xi
$$

that is $\operatorname{det}\left(I-R_{s 1}\right)=0$, where $R_{s t}$ is the solution of $\dot{R}=J S(s) R, R_{s 0}=I$. It is known ([8] Section 17, [43] Thm. A) that the Fredholm index of $F$ is the spectral flow of the family $(A(s))$ when $s$ goes from $-\infty$ to $+\infty$, that is the algebraic number of eigenvalues crossing 0 . Finally ind $(F)$ is the algebraic number of crossings of $\left(R_{s 1}\right)_{-\infty}^{+\infty}$ with the Maslov cycle when it is endowed with a suitable co-orientation (cf. Appendix). It is "clear" from Figure 8 that this number is the desired one $\mu\left(\left(R_{t}^{+}\right)\right)-\mu\left(\left(R_{t}^{-}\right)\right)$.

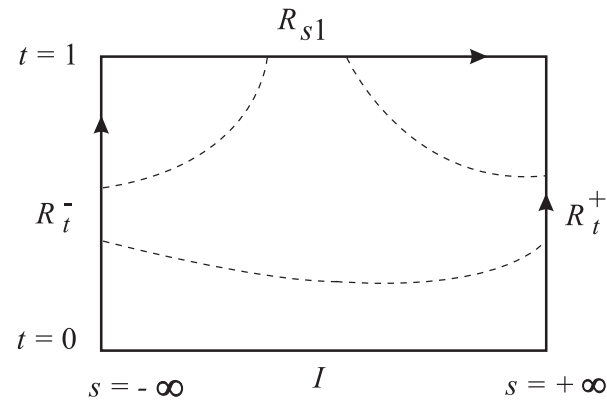

Figure 8: The dot lines are $\left\{(s, t) \mid \operatorname{det}\left(I-R_{s, t}\right)=0, t>0\right\}$.

Of course one needs to be careful along $t=0$. But the index of $F$ depends only on the path $\left(R_{s 1}\right)$ and the Conley-Zehnder indices of the vertical sides 
do not change if their endpoints are kept fixed. This remark allows us to take $S(s, t)$ invertible for all $s$ and small $t$. In that case $\{t=0\}$ becomes isolated in $\left\{\operatorname{det}\left(I-R_{s t}\right)=0\right\}$ and Figure 8 tells us the truth.

\subsection{The Conley-Zehnder index of a 1-periodic orbit}

Let us consider $(M, \omega, H)$ as in theorem 5.1. It is possible to define $i_{\mathrm{CZ}}(x)$ when $x$ is a non-degenerate 1-periodic orbit of the Hamilton equation. We choose an almost complex structure $J$ which is $\omega$-compatible. The complex fiber bundle $\left(x^{*} T M, J\right)$ is trivial and, in fact, this trivialization is unique up to homotopy. Indeed, according to 2) from theorem 5.1, it does not depend on the contraction chosen for $x$. Having trivialized $\left(x^{*} T M, J\right)$, the linearized differential equation reads as in the $\mathbb{R}^{2 n}$ - case and $i_{C Z}(x)$ is defined as before.

\subsection{Application to the gradient lines of the action}

Notations. For a 1-periodic Hamiltonian $H, P_{H}$ will denote the set of contractible 1-periodic solutions of the Hamilton equation $\dot{x}=X_{H}(t, x)$. By assumption they are all non-degenerate. We have

$$
P_{H}=\operatorname{crit} \Phi_{H}
$$

where $\Phi_{H}$ is the action functional defined on the smooth contractible loops. For $x_{-}, x_{+} \in P_{H}, \mathcal{M}\left(x_{-}, x_{+}\right)$will denote the set of gradient lines descending from $x_{-}$to $x_{+}$. That means:

$$
\left\{\begin{array}{l}
u \in \mathcal{M}\left(x_{-}, x_{+}\right) \Longleftrightarrow \\
u: \mathbb{R} \times S^{1} \rightarrow T^{2 n} \text { is smooth } \\
\frac{\partial u}{\partial s}+J(u) \frac{\partial u}{\partial t}+\nabla H(t, u)=0 \\
u \rightarrow x_{ \pm} \text {if } s \rightarrow \pm \infty
\end{array}\right.
$$

\section{Remark 5.7. About the analytic setting.}

The general aim is to describe the set $\mathcal{M}\left(x_{-}, x_{+}\right)$as a submanifold of some Banach manifold on which the perturbed Cauchy-Riemann equation would be a regular Fredholm equation. This can be achieved when working with $W^{1, p}$, $p>2$, the space of functions in $L^{p}$ whose first derivatives are in $L^{p}$. When the source is 2-dimensional, such a function is continuous (it can be non-continuous if $p=2$ ). As we do not intend to deal with this sort of difficulty, in the sequel we shall restrict ourselves to the case where $M$ is the $2 n$-torus as a differentiable manifold; as it is said below, in that case it is sufficient to work with $W^{1,2}$. But except for the Sobolev setting we will argue as in the general case. 
Indeed, when $M=T^{2 n}=\mathbb{R}^{2 n} / \mathbb{Z}^{2 n}$ and if $\pi: \mathbb{R}^{2 n} \rightarrow T^{2 n}$ denotes the projection, it is easy to define $W^{1,2}\left(\mathbb{R} \times S^{1}, M, x_{-}, x_{+}\right)$; it is a set of $u$ 's of the form $u=\pi \circ \tilde{u}$, where $\tilde{u}$ belongs to $W_{\text {loc }}^{1,2}\left(\mathbb{R} \times S^{1}, \mathbb{R}^{2 n}\right)$ with some limit conditions at infinity we are explaining now. First we recall for $\tilde{u} \in W_{\text {loc }}^{1,2}\left(\mathbb{R} \times S^{1}, \mathbb{R}^{2 n}\right)$, according to the Fubini theorem, for almost all $s \in \mathbb{R}$ the restriction $\tilde{u}(s)=\tilde{u}_{\mid\{s\} \times S^{1}}$ lies in $W^{1,2}\left(S^{1}, \mathbb{R}^{2 n}\right)$, which is a space of continuous functions; the corresponding $u(s): S^{1} \rightarrow T^{2 n}$ is continuous accordingly. This enables us to define $W^{1,2}\left(\mathbb{R} \times S^{1}, T^{2 n}, x_{-}, x_{+}\right)$by the following requirements: there is some lifting $\tilde{x}_{ \pm}$of $x_{ \pm}$to $\mathbb{R}^{2 n}$ such that $\tilde{u}-\tilde{x}_{ \pm}$belongs to $W^{1,2}\left(\mathbb{R}_{ \pm} \times S^{1}, \mathbb{R}^{2 n}\right)$. It makes sense because the loop $x_{ \pm}$is homotopic to zero.

As a consequence, for almost every $t \in S^{1}, \tilde{u}(s, t)-\tilde{x}_{ \pm}(t)$ is continuous and tends to 0 when $s$ goes to $\pm \infty$ ([10] corollaire VIII.8). Therefore the lifting $x_{ \pm}$is uniquely determined by the choice of $\tilde{u}$ representing $u$; in particular the choice of $\tilde{u}$ is unique up to covering transformation. Now it is clear that $W^{1,2}\left(\mathbb{R} \times S^{1}, T^{2 n}, x_{-}, x_{+}\right)$is a Banach manifold modelled on $W^{1,2}\left(\mathbb{R} \times S^{1}, \mathbb{R}^{2 n}\right)$.

Proposition 5.8. $\mathcal{M}\left(x_{-}, x_{+}\right)$is defined by a Fredholm equation in the sense that, at every $u \in \mathcal{M}\left(x_{-}, x_{+}\right)$, the linearized equation $F_{u} \xi=0$ is Fredholm. Moreover the index of $F_{u}$ is

$$
\operatorname{ind}\left(F_{u}\right)=i_{C Z}\left(x_{-}\right)-i_{C Z}\left(x_{+}\right) .
$$

Proof. For $u \in \mathcal{M}\left(x_{-}, x_{+}\right)$and $\xi$, a vector field along $u$ tangent to $T^{2 n}$,

$$
F_{u} \xi:=D_{s} \xi+J(u) D_{t} \xi+\left(D_{\xi} J(u)\right) \cdot \frac{\partial u}{\partial t}+D_{\xi}(\nabla H(t, u))
$$

where $D_{s}$ and $D_{t}$ stand for the covariant derivative with respect to $\frac{\partial u}{\partial s}$ and $\frac{\partial u}{\partial t}$ respectively. Let $R=\left(Z_{1}, \ldots, Z_{2 n}\right)$ be an orthonormal $J$-invariant framing of $u^{*}\left(T\left(T^{2 n}\right)\right)$ (that is $J R=R$ up to a permutation) which extends over the limit orbits $x_{+}$and $x_{-}$. If $\xi=R \zeta$, with $\zeta(s, t) \in \mathbb{R}^{2 n}$, we get

$$
\left(R^{*} F_{u}\right) \zeta=\frac{\partial \zeta}{\partial s}+J \frac{\partial \zeta}{\partial t}+L . \zeta
$$

where $L . \zeta$ is an operator of order 0 , that is $L(s, t) \in \operatorname{End}\left(\mathbb{R}^{2 n}\right)$.

Claim. When $s$ goes to $\pm \infty$, the limit of $L(s, t)$ is a symmetric matrix.

Proof of the claim. The $(i, j)$-entry of $L(s, t)$ is given by the value at $(s, t)$ of the following sum:

$$
<Z_{i}, D_{s} Z_{j}+J D_{t} Z_{j}+D_{Z_{j}}(J(u)) \cdot \frac{\partial u}{\partial t}+D_{Z_{j}} \nabla H>
$$


According to corollary 4.9 the limit when $s \rightarrow \pm \infty$ is

$$
\ell_{i j}^{ \pm}(t):=<Z_{i}, J D_{t} Z_{j}+D_{Z_{j}}(J) \cdot \dot{x}_{ \pm}+D_{Z_{j}} \nabla H>_{\mid x_{ \pm}(t)} .
$$

From $\dot{x}_{ \pm}=J\left(x_{ \pm}\right) \nabla H\left(t, x_{ \pm}\right)$and $J . D_{Z_{j}}(J)=-D_{Z_{j}}(J) . J$ we derive :

$$
\begin{aligned}
\ell_{i j}^{ \pm}(t) & =<Z_{i}, J\left(D_{X} Z_{j}-D_{Z_{j}} X\right)> \\
& =<Z_{i}, J\left[X, Z_{j}\right]>=-\omega\left(Z_{i},\left[X, Z_{j}\right]\right),
\end{aligned}
$$

where $X:=J \nabla H$. Since $X$ is a Hamiltonian vector field its flow preserves $\omega$. Then

$$
\begin{aligned}
0 & =X . \omega\left(Z_{i}, Z_{j}\right) \\
& =\left(L_{X} \omega\right)\left(Z_{i}, Z_{j}\right)+\omega\left(\left[X, Z_{i}\right], Z_{j}\right)+\omega\left(Z_{i},\left[X, Z_{j}\right]\right) \\
& =\omega\left(Z_{i},\left[X, Z_{j}\right]\right)-\omega\left(Z_{j},\left[X, Z_{i}\right]\right)
\end{aligned}
$$

hence $L$ is symmetric.

As a consequence the linearized equation $\left(R^{*} F_{u}\right) \zeta=0$ takes the form required in 5.2. From theorem 5.6 we deduce that

$$
R^{*} F_{u}: W^{1,2}\left(\mathbb{R} \times S^{1}, \mathbb{R}^{2 n}\right) \rightarrow L^{2}\left(\mathbb{R} \times S^{1}, \mathbb{R}^{2 n}\right)
$$

is a Fredholm operator and its index is $i_{C Z}\left(x_{-}\right)-i_{C Z}\left(x_{+}\right)$.

In general the Fredholm property is not sufficient in order to deduce that $\mathcal{M}\left(x_{-}, x_{+}\right)$is a finite dimensional manifold. But we observe that $H$ and $J$ play the role of parameters in the equation of $\mathcal{M}\left(x_{-}, x_{+}\right)$.

Definition 5.9. $(H, J)$ is a regular pair if, for every $u \in \mathcal{M}\left(x_{-}, x_{+}\right), F_{u}$ is surjective.

It is easy to prove that there are enough possible deformations of $(H, J)$ that for a pair to be regular is a generic property in Baire's sense. This fact follows from the infinite dimensional version of Sard's theorem due to Smale, which applies to $\left(C^{\infty}\right)$ - nonlinear Fredholm maps between Banach spaces ([51]).

Remark 5.10. If we start with a Hamiltonian function $H$ whose 1-periodic solutions are non-degenerate and with an arbitrary $\omega$-compatible almost complex structure $J$, then approximating $(H, J)$ by a regular pair $(\widetilde{H}, \widetilde{J})$ close enough to $(H, J)$ does not change the number of 1-periodic solutions nor their indices since they can be followed along the approximation, according to the implicit function theorem (compare subsection 4.3). Moreover I think it is possible to take $\widetilde{H}=H$, but after the previous remark this question is irrelevant for theorem 5.1 .

Corollary 5.11. When $(H, J)$ is regular, $\mathcal{M}\left(x_{-}, x_{+}\right)$is a finite dimensional manifold, whose dimension is $i_{C Z}\left(x_{-}\right)-i_{C Z}\left(x_{+}\right)$. 
Proof. Apply the implicit function theorem.

As in Morse theory we have a natural right action of $\mathbb{R}$ onto the manifold $\mathcal{M}\left(x_{-}, x_{+}\right)$by translation of the variable $s$. In particular when $i_{C Z}\left(x_{-}\right)-$ $i_{C Z}\left(x_{+}\right)=0, \mathcal{M}\left(x_{-}, x_{+}\right)$is empty since if it contains a point it would contain a line.

Theorem 5.12. There exists a natural compactification of $\mathcal{M}\left(x_{-}, x_{+}\right) / \mathbb{R}$ made of adding the broken $L^{2}$-gradient lines from $x_{-}$to $x_{+}$. It is a manifold (with boundary and corners) of dimension $i_{C Z}\left(x_{-}\right)-i_{C Z}\left(x_{+}\right)-1$.

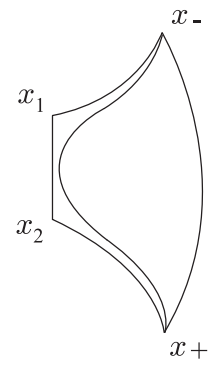

Figure 9: A broken $L^{2}$-gradient line with two vertices.

In the example of figure 9 we have $i_{C Z}\left(x_{-}\right)>i_{C Z}\left(x_{1}\right)>i_{C Z}\left(x_{2}\right)>i_{C Z}\left(x_{+}\right)$. Sometimes this theorem is called the glueing theorem and it corresponds to a piece of work in Floer's theory.

Corollary 5.13. If $i_{C Z}\left(x_{-}\right)=i_{C Z}\left(x_{+}\right)+1, \mathcal{M}\left(x_{-}, x_{+}\right) / \mathbb{R}$ is a compact 0 dimensional manifold and hence a finite set whose cardinality mod 2 is denoted by $n\left(x_{-}, x_{+}\right)$.

\subsection{The Floer complex}

Given a regular pair $(H, J)$, the vector spaces of the Floer complex are:

$$
C F_{k}(H, J)=\underset{x \in P(H), i_{C Z}(x)=k}{\oplus}(\mathbb{Z} / 2 \mathbb{Z}) \cdot x
$$

The boundary operator $\partial_{k}: C F_{k}(H, J) \rightarrow C F_{k-1}(H, J)$ is defined by:

$$
\partial_{k}(x)=\sum_{i_{C Z}(y)=k-1} n(x, y) y .
$$




\section{Theorem 5.14.}

$$
\partial_{k} \circ \partial_{k+1}=0
$$

Using theorem 5.12, the proof of theorem 5.14 goes as in Morse theory. This allows us to define homology groups

$$
H F_{k}(H, J)=\frac{\operatorname{ker} \partial_{k}}{\operatorname{im} \partial_{k+1}} .
$$

The following theorem claims that the Floer homology does not depend on the chosen regular pair $(H, J)$. It is similar to theorem 3.10 .

Theorem 5.15. If $\left(H_{0}, J_{0}\right)$ and $\left(H_{1}, J_{1}\right)$ are two regular pairs then there exists a canonical isomorphism

$$
H F_{*}\left(H_{0}, J_{0}\right) \rightarrow H F_{*}\left(H_{1}, J_{1}\right) .
$$

The last result we will mention and which implies theorem 5.1 is the following.

Theorem 5.16. Let $H$ be a Morse function independent of time and $J$ an almost complex structure $\omega$-compatible. We assume the Smale transversality condition: for the Riemannian metric $\langle\xi, \eta\rangle=\omega(\xi, J \eta)$ the stable and unstable manifolds are transversal in $M$.

Then, for $\tau \in] 0,1]$ small enough, the following holds:

1) $(\tau H, J)$ is regular.

2) $H F_{*}(\tau H, J) \cong H_{*+n}(M, \mathbb{Z} / 2 \mathbb{Z})$.

Proof. We can start with $H$ so $C^{2}$-small that lemma 2.2 applies. Then the only 1-periodic orbits of $\dot{x}=J \nabla H(x)$ are the critical points of $H$. In that case the linearized Hamilton equation at a critical point $x_{0}$ is

$$
\dot{\xi}=J D^{2} H\left(x_{0}\right) \xi .
$$

If $\left\|D^{2} H\left(x_{0}\right)\right\|<2 \pi$ the Conley-Zehnder index is $i_{C Z}\left(x_{0}\right)=\operatorname{ind}\left(x_{0}\right)-n$. Let $x_{-}$and $x_{+}$be two critical points of $H$ with $i_{C Z}\left(x_{-}\right)=i_{C Z}\left(x_{+}\right)+1$. We have to find all the solutions of

$$
\frac{\partial u}{\partial s}+J(u) \frac{\partial u}{\partial t}+\nabla H(u)=0
$$

such that $u \rightarrow x_{ \pm}$when $s$ goes to $\pm \infty$. For this purpose we follow SalamonZehnder ([45], p. 1342).

Lemma 5.17. There exists $\tau_{0}>0$ such that, for every $\tau \leq \tau_{0}$, any $u \in$ $\mathcal{M}\left(x_{-}, x_{+}\right)$, which is $\tau$-periodic in $t$, is independent of $t$. 
Proof. If this is not true, there exists a sequence $u_{k} \in \mathcal{M}\left(x_{-}, x_{+}\right)$where $u_{k}$ is periodic in $t$ with a minimal period $\tau_{k}$ such that $0<\tau_{k} \leq 1 / k$. According to the broken connecting orbit theorem 4.10 , taking a subsequence and a suitable shift of $s$ if necessary, $u_{k}$ converges to a piece $u \in \mathcal{M}$ of a broken connecting orbit $\gamma$ from $x_{-}$to $x_{+}$. Obviously $u$ (and all pieces of $\gamma$ as well) is 0-periodic in $t$ and hence it is a solution of

$$
\frac{\partial u}{\partial s}+\nabla H(u)=0
$$

that is a usual gradient line of $H$ in $M$. Under the Smale tranversality condition, there are no usual broken connecting orbits from $x_{-}$to $x_{+}$in $M$ which are really broken, because their Morse indices differ by 1 . As a consequence $u_{k} \rightarrow$ $u \in \mathcal{M}\left(x_{-}, x_{+}\right)$which is a usual gradient line. For such an $u$, there are two linearized operators. The first one $F_{u}^{0}$ is obtained in linearizing equation (1) of gradient lines of $H$. The second one $F_{u}^{\tau}$, depending on a parameter $\tau$, is obtained in linearizing the Cauchy-Riemann equation

$$
\frac{\partial u}{\partial s}+J(u) \frac{\partial u}{\partial t}+\nabla H(u)=0
$$

where $u$ is $\tau$-periodic in $t$, that is $u: \mathbb{R} \times \mathbb{R} / \tau \mathbb{Z} \rightarrow M$ and $x_{ \pm}=\lim _{s \rightarrow \pm \infty} u(s, t)$.

Let us be more precise. As in proposition 5.8 we use a framing $R$ of $u^{*} T M$. Because $u$ does not depend on $t$, one can choose $R$ so that it does not either. The equation $F_{u}^{\tau} \xi=0$ becomes

$$
\left(R^{*} F_{u}^{\tau}\right) \zeta:=\frac{\partial \zeta}{\partial s}+J \frac{\partial \zeta}{\partial t}+S(s) \zeta=0
$$

for $\zeta: \mathbb{R} \times \mathbb{R} / \tau \mathbb{Z} \rightarrow \mathbb{R}^{2 n}$. The equation $F_{u}^{0} \xi=0$ becomes

$$
\left(R^{*} F_{u}^{0}\right) \zeta:=\frac{d \zeta}{d s}+S(s) \zeta=0
$$

for $\zeta: \mathbb{R} \rightarrow \mathbb{R}^{2 n}$. Here $S(s)$ is an endomorphism of $\mathbb{R}^{2 n}$ having a limit at infinity (indeed a symmetric endomorphism).

\section{Sublemma.}

1) $F_{u}^{0}: W^{1,2}\left(\mathbb{R}, u^{*} T M\right) \rightarrow L^{2}\left(\mathbb{R}, u^{*} T M\right)$ is surjective and its Fredholm index $i s \operatorname{ind}\left(x_{-}\right)-\operatorname{ind}\left(x_{+}\right)$.

If $\tau$ is small enough, the two following conclusions hold:

2) $\operatorname{ker} F_{u}^{0}=\operatorname{ker} F_{u}^{\tau}$.

3) $F_{u}^{\tau}$ is surjective. 


\section{References for proofs}

1) This is proven with all details in [44], thm. 3.3.

2) For $\zeta: \mathbb{R} \times \mathbb{R} / \tau \mathbb{Z} \rightarrow \mathbb{R}^{2 n}$, let $\mu(\zeta)$ be its mean with respect to the $t$-variable. On the one hand, $\left(R^{*} F_{u}^{\tau}\right) \zeta=0$ yields $\left(R^{*} F_{u}^{0}\right) \mu(\zeta)=0$. On the other hand if $\mu(\zeta)=0$, there is an elementary estimate

$$
\|\zeta\|_{L^{2}} \leq \tau\left\|\frac{\partial \zeta}{\partial t}\right\|_{L^{2}}
$$

from which one can derive :

$$
\left\|\frac{\partial \zeta}{\partial t}\right\|_{L^{2}} \leq\left\|\left(R^{*} F_{u}^{\tau}\right) \zeta\right\|_{L^{2}}+c \tau\left\|\frac{\partial \zeta}{\partial t}\right\|_{L^{2}}
$$

where $c$ is a uniform bound of $\|S(s)\|$ (compare [45], p. 1327-1328). When $\tau$ is small enough, we have $c \tau<1$. In that case $\left(R^{*} F_{u}^{\tau}\right) \zeta=0$ and $\mu(\zeta)=0$ imply $\zeta=0$; or: $\left(R^{*} F_{u}^{\tau}\right) \zeta=0$ implies $\zeta=\mu(\zeta)$, which is independent of $t$. Finally $\xi \in \operatorname{ker} F_{u}^{\tau}$ implies $\xi \in \operatorname{ker} F_{u}^{0}$. The converse being obvious we have the wanted conclusion.

3) According to 1) we have

$$
\begin{aligned}
\operatorname{dim} \operatorname{ker} F_{u}^{0} & =\operatorname{ind}\left(x_{-}\right)-\operatorname{ind}\left(x_{+}\right) \\
& =i_{C Z}\left(x_{-}\right)-i_{C Z}\left(x_{+}\right) \\
& =\operatorname{ind}\left(F_{u}^{\tau}\right) .
\end{aligned}
$$

According to 2) it is also the dimension of $\operatorname{ker} F_{u}^{\tau}$. Thus $F_{u}^{\tau}$ is surjective.

We return to the proof of lemma 5.17. Let us fix some $\tau$ such that points 2) and 3$)$ in the above sublemma are valid. Since the Fredholm index of $F_{u}^{\tau}$ is 1 , by the implicit function theorem, $u$ is isolated in $\mathcal{M}_{\tau}\left(x_{-}, x_{+}\right)$up to a shift in $s$, where $\mathcal{M}_{\tau}\left(x_{-}, x_{+}\right)$is the space of solutions of the perturbed Cauchy Riemann equation, which are $\tau$-periodic in time (it makes sense even if $\tau$ is not a divisor of 1 , because the Hamiltonian is autonomous) and whose limits at infinity are respectively $x_{-}$and $x_{+}$. Moreover $u$ is uniformly isolated in $\mathcal{M}_{\tau^{\prime}}\left(x_{-}, x_{+}\right)$for all $\tau^{\prime}$ close enough to $\tau$, let us say $\left.\tau^{\prime} \in\right] \tau-\varepsilon, \tau+\varepsilon$ [; for that we use the implicit function theorem with a parameter. When $k$ is large, the period $\tau_{k}$ is small, some integer multiple $\tau_{k}^{\prime}$ of $\tau_{k}$ lies in $] \tau-\varepsilon, \tau+\varepsilon$ [ and $u_{k}$ is close to $u$. Hence $u$ is not isolated in $\mathcal{M}_{\tau_{k}^{\prime}}\left(x_{-}, x_{+}\right)$, leading to a contradiction. So the lemma is proven. 
We now finish the proof of theorem 5.16. Let us consider the Hamiltonian $\tau H$ and $u_{\tau}(s, t):=u(\tau s, \tau t)$. One checks easily that:

1. $u_{\tau}$ is 1-periodic in $t$ iff $u$ is $\tau$-periodic,

2. $u_{\tau}$ is a solution of the Cauchy-Riemann equation perturbed by $\tau H$ with $x_{ \pm}$as boundary condition iff $u \in \mathcal{M}\left(x_{-}, x_{+}\right)$.

3. $F_{u_{\tau}}$ is surjective iff $F_{u}^{\tau}$ is surjective.

Let $u_{\tau}$ be a 1-periodic solution of

$$
\frac{\partial u}{\partial s}+J(u) \frac{\partial u}{\partial t}+\nabla \tau H(u)=0
$$

with $x_{ \pm}$as boundary conditions. Let $u$ be the associated $\tau$-periodic map as above. According to the sublemma, if $\tau$ is small enough the pair $(\tau H, J)$ is regular.

In choosing $\tau_{0}$ so that lemma 5.17 is valid, we deduce that $u$ is 0 -periodic, hence $u_{\tau}$ also is. In other words the only 1-periodic solutions of

$$
\frac{\partial u}{\partial s}+J(u) \frac{\partial u}{\partial t}+\nabla \tau H(u)=0
$$

with $x_{ \pm}$as boundary conditions are the solutions of

$$
\frac{\partial u}{\partial s}+\nabla \tau H(u)=0
$$

that is the usual gradient lines from $x_{-}$to $x_{+}$. This amounts to saying that the Floer complex $C F_{*}(\tau H, J)$ coincides with the Morse complex up to a translation of the grading.

Corollary 5.18. Let us consider the same data and assumptions as in theorem 5.1. Let us denote by $p_{k}$ the number of 1-periodic orbits with Conley-Zehnder index $k$ and by $b_{k}$ the $k^{\text {th }}$ Betti number over $\mathbb{Z} / 2 \mathbb{Z}$. The Morse inequalities are valid:

$$
p_{k}-p_{k-1}+\ldots \geq b_{n+k}-b_{n+k-1}+\ldots+(-1)^{k+n} b_{0} .
$$

\section{Final comments}

There are many other problems where a sort of Floer homology or cohomology is involved; one speaks of cohomology when a product is defined and used. Such groups, even when there are well defined, are not always computable. It is the same situation as in classical algebraic topology; as there, even if $H F_{*}$ or $H F^{*}$ is not computable, some deep results can be deduced for instance in arguing with 
exact sequences. Here are a few examples where various Floer (co)-homologies appear.

1) Fixed points of symplectic diffeomorphisms which are not Hamiltonian (see [16]). In [47] P. Seidel succeeded in computing the Floer homology of a Dehn twist along a multi-curve on a surface.

2) Intersections of Lagrangian submanifolds [21]. Here we have to recall that this problem (and paper) is the historical one because it is the first which has been solved by means of Floer homology. In fact the problem of fixed points of symplectic diffeomorphisms could be regarded as a particular case of this one: take the graph of the diffeomorphism under consideration and look at its intersection with the diagonal. Due to the topological restrictions in [21], Floer found more efficient to create another Floer homology for the fixed points problem in [24].

3) Picard-Lefschetz theory. Of particular interest is the generalization of the 2-dimensional Dehn twist discovered by V.I. Arnold in high dimension, namely the Dehn twist along a Lagrangian sphere [5]. It appears as the monodromy diffeomorphism of a complex Morse singularity. P. Seidel formulated a symplectic analogue of the holomorphic Morse functions and revealed a long exact sequence involving the Floer homology of a pair of exact Lagrangian submanifolds and the Dehn twist along an exact Lagrangian sphere [48].

4) The Weinstein conjecture [57]: Any closed hypersurface $\Sigma$ of contact type in a symplectic manifold $(M, \omega)$ carries a closed characteristic (contact type means that along $\Sigma$ there is a transversal vector field dilating $\omega$ ). In complete generality it is still an open question. The linear case $(M, \omega)=\left(\mathbb{R}^{2 n}, \omega_{0}\right)$ was proven by C. Viterbo [55]; the proof was based on a linking phenomenon in some loop space. Since this time many other cases have been solved by means of various tools including pseudoholomorphic techniques. In [56] Viterbo succeeded in putting the Weinstein conjecture into the Floer homology world. It allowed him to recover the known cases and to solve new ones like: $M=T^{*} N$ where $N$ is 1-connected, or $M$ is a sub-critical Stein manifold.

5) The "chord problem" for Legendrian submanifolds in a contact manifold. In the case of a Legendrian knot $k$ in $\mathbb{R}^{3}$ endowed with its standard contact form $\alpha=d z-y d x$, Y. Chekanov [13] gave a "combinatorial" Floer homology related to the chords of $k$, that is to the double points of the $x y$-projection of $k$. In that way he succeeded in discovering Legendrian knots with the same topological type and Thurston-Bennequin invariant, but which are not contact isotopic. In [20] the Chekanov complex is related to a Floer complex of $J$ holomorphic curves in the symplectization $\left(\mathbb{R}^{3} \times \mathbb{R}, \omega=d\left(e^{\tau} \alpha\right)\right)$ in the spirit of the contact homology theory from [19].

6) More about contact structures in dimension 3. P. Ozváth and Z. Szabó invented a Heegaard-Floer homology for 3-manifolds equipped with a Spin ${ }^{c}$ structure [39]. When a contact structure $\xi$ (and the associated $\operatorname{Spin}^{c}$-structure) 
is given on the 3-manifold $Y$, they define an integer $c(\xi)$ coming from calculations of some Heegaard-Floer groups. This invariant is non trivial but vanishes when $\xi$ is overtwisted. So it is a deep new tool for studying tight contact structures. 


\section{Appendix : Topology of $\operatorname{Sp}(2 n)$}

\section{A1 Local properties}

We consider $\mathbb{R}^{2 n}=\mathbb{C}^{n}$ endowed with its standard symplectic structure $\omega_{0}$ and its complex structure $J$. The basis is ordered as a real basis of $\mathbb{C}^{n}=(\mathbb{R}+$ $i \mathbb{R})^{n}$. The linear symplectic group $\operatorname{Sp}(2 n)$ is the group of linear isomorphisms of $\left(\mathbb{R}^{2 n}, \omega_{0}\right)$. It is identified with a group of invertible $2 n \times 2 n$ matrices. For $A \in \mathrm{Sp}(2 n)$ close to $I$, one can write $A=e^{J S}$, where $S$ is symmetric. Then $\operatorname{Sp}(2 n)$ is a Lie group which has the same dimension as the space of quadratic forms on $\mathbb{R}^{2 n}$, that is $n(2 n+1)$. We recall notation from section 5 :

the Maslov cycle $\Sigma:=\{A \in \operatorname{Sp}(2 n) \mid \operatorname{det}(I-A)=0\}$, and $\operatorname{Sp}^{*}(2 n):=\{A \in \operatorname{Sp}(2 n) \mid \operatorname{det}(I-A) \neq 0\}$.

The Maslov cycle is stratified by the dimension of $V_{1}(A)$, where $V_{1}(A)$ denotes the eigenspace of $A$ of the eigenvalue 1: $\Sigma=\Sigma_{1} \sqcup \Sigma_{2} \sqcup \cdots \sqcup \Sigma_{2 n}$, where the union is made of disjoint strata

$$
\Sigma_{k}=\operatorname{Sp}_{k}(2 n):=\left\{A \in \operatorname{Sp}(2 n) \mid \operatorname{dim} V_{1}(A)=k\right\} .
$$

Since $A \mapsto \operatorname{dim}\left(V_{1}(A)\right)$ is semi-continuous, the closure of $\Sigma_{k}$ is $\bar{\Sigma}_{k}=\Sigma_{k} \cup$ $\Sigma_{k+1} \cdots \cup \Sigma_{2 n}$. We recall that a linear isomorphim $A$ of $\mathbb{R}^{2 n}$ is symplectic iff its graph $\Gamma_{A}$ in $\mathbb{R}^{2 n} \times \mathbb{R}^{2 n}$ is Lagrangian with respect to $\omega_{0} \ominus \omega_{0}$. The intersection of $\Gamma_{A}$ with the diagonal $\Delta$ projects onto $V_{1}(A)$.

For a vector space $E, \operatorname{Sym}(E)$ denotes the space of self-adjoint operators $S$ : $E \rightarrow E^{*}$ and $\operatorname{Sym}_{k}(E)$ denotes the subspace of those whose kernel has dimension $k$.

\section{Proposition A1.1.}

1) $\Sigma_{k}$ is a smooth submanifold of codimension $\frac{k(k+1)}{2}$ in $\operatorname{Sp}(2 n)$.

2) Let $A_{0} \in \Sigma_{k}$ and $E_{0}:=\Gamma_{A_{0}} \cap \Delta$. There exists a germ of smooth embedding

$$
T:\left(\operatorname{Sym}\left(E_{0}\right), 0\right) \rightarrow\left(\operatorname{Sp}(2 n), A_{0}\right)
$$

with the following properties:

i) $T$ is transversal to $\Sigma_{k}$ at $A_{0}$.

ii) $T(s) \in \Sigma_{j} \Longleftrightarrow s \in \operatorname{Sym}_{j}\left(E_{0}\right)$.

Proof. 1) In order to study $\operatorname{Sp}(2 n)$ near $A_{0}$ we choose a Lagrangian complement $\Delta^{*}$ of the diagonal $\Delta$ transversal to $\Delta$ and to the graph $\Gamma_{A_{0}}$. It is indeed canonically isomorphic to the dual of $\Delta$. Every Lagrangian subspace in $\mathbb{R}^{2 n} \times$ $\mathbb{R}^{2 n}$ close to $\Gamma_{A_{0}}$ is a $\Gamma_{A}$ for some $A \in \operatorname{Sp}(2 n)$.

Let $A_{0}$ be in $\Sigma_{k}$. A nearby $A$ lies in $\Sigma_{k}$ iff $\operatorname{dim}\left(\Gamma_{A} \cap \Delta\right)=k$. Let $\mathcal{L}$ be the space of Lagrangian subspaces in $\Delta \oplus \Delta^{*}$ transversal to $0 \oplus \Delta^{*}$. Let us denote

$$
\mathcal{L}_{k}:=\{L \in \mathcal{L} \mid \operatorname{dim}(L \cap \Delta)=k\} .
$$


Every $L \in \mathcal{L}$ has a generating quadratic form $q$. Precisely, $L$ is the graph of a linear self-adjoint operator $B: \Delta \rightarrow \Delta^{*}$ and $q(x)=\langle B(x), x>$. Let us split $\Delta$ into $\Delta=E_{0} \oplus E_{0}^{\prime}$ and dually $\Delta^{*}=E_{0}{ }^{*} \oplus E_{0}^{\prime *}$. Then $B$ reads as a matrix of operators

$$
B=\left(\begin{array}{cc}
a & { }^{t} b \\
b & c
\end{array}\right)
$$

where $a$ and $c$ are self-adjoint. For $L_{0}:=\Gamma_{A_{0}}$, the corresponding matrix is

$$
B_{0}=\left(\begin{array}{cc}
0 & 0 \\
0 & c_{0}
\end{array}\right)
$$

where $c_{0}$ is invertible. When $B$ is close to $B_{0}, c$ is also invertible and

$$
L \in \mathcal{L}_{k} \Longleftrightarrow a+{ }^{t} b c^{-1} b=0 \quad(*) .
$$

The last equation says that the kernel of $B$ has dimension $k$ (and not less). Since this equation is regular near $B_{0}$, we have proven 1 ). Moreover, in these coordinates, the equation of the tangent space $T_{L_{0}}\left(\mathcal{L}_{k}\right)$ reads $a=0$.

2) As a consequence of the last remark the affine space of generating quadratic forms

$$
\left(\begin{array}{cc}
a & 0 \\
0 & c_{0}
\end{array}\right)
$$

is transversal to $\mathcal{L}_{k}$ at $\Gamma_{A_{0}}$. Therefore when $a$ is so small that $L$ is a $\Gamma_{A}$, the corresponding family of $A$ 's is transversal to $\Sigma_{k}$ at $A_{0}$. It is the transversal $T$ we are looking for. Moreover, the dimension of the kernel of matrix $(* *)$ is equal to the dimension of ker $a$. Then $2 \mathrm{ii}$ ) is clear.

From the above properties we immediatly deduce the following corollary.

\section{Corollary A1.2.}

1) The codimension of $\Sigma_{k}$ in $\operatorname{Sp}(2 n)$ is at least 3 when $k>1$.

2) $\bar{\Sigma}_{2}$ locally separates the Maslov cycle.

3) $\Sigma \backslash \Sigma_{3}$ is locally connected.

\section{A2. Global connectivity properties}

Lemma A2.1. $\Sigma_{1} \cup \Sigma_{2}$ is arcwise-connected.

Proof. According to 3 ) from corollary A1.2, it is sufficient to prove that $\Sigma$ is arcwise connected.

Let $A_{1}$ and $A_{2}$ be two symplectic isomorphisms with 1 as eigenvalue. Let us choose $v_{1}$ and $v_{2}$ two eigenvectors of $A_{1}$ and $A_{2}$ respectively. Because $\operatorname{Sp}(2 n)$ acts transitively on $\mathbb{R}^{2 n} \backslash 0$ one can move $A_{2}$ in $\Sigma$ by means of a 1-parameter family of conjugations in order to reduce ourselves to $v_{2}=v_{1}=: v$. The subgroup $\operatorname{Sp}(2 n)_{v}$ stabilizing $v$ acts transitively on the set of Lagrangian subspaces 
containing $v$. Then we can move $A_{1}$ and $A_{2}$ in $\operatorname{Sp}(2 n)_{v}$ such that they share a common invariant Lagrangian subspace $L$. The subgroup $\operatorname{Sp}(2 n)_{L}$ stabilizing $L$ acts transitively on the set of Lagrangian subspaces transversal to $L$. Then we are reduced to the case where $A_{1}$ and $A_{2}$ keep invariant two transversal Lagrangian subspaces $L$ and $L^{*}$, with $v \in L$. In that case there are a basis $B$ of $L$, containing $v$, and its dual basis $B^{*}$ of $L^{*}$ such that, for $i=1,2, A_{i}$ reads in $\left(B, B^{*}\right)$ as

$$
A_{i}=\left(\begin{array}{cccc}
1 & \times & & 0 \\
0 & \Phi_{i} & & \\
0 & 1 & 0 \\
& & \times & { }^{t} \Phi_{i}^{-1}
\end{array}\right)
$$

Now connecting $A_{1}$ and $A_{2}$ in $\Sigma$ is an exercise which is left to the reader whatever the signs of $\operatorname{det} \Phi_{i}$ may be.

Proposition A2.2. $\Sigma_{0}:=\operatorname{Sp}(2 n) \backslash \Sigma$ has two connected components.

Proof. One already knows that there are at least two components, one for each sign of $\operatorname{det}(I-A)$. Because $\operatorname{Sp}(2 n)$ is connected, the closure of each component of $\Sigma_{0}$ meets $\Sigma_{1}$. Hence it is sufficient to prove that, for every path $\gamma:[0,1] \rightarrow \Sigma_{1} \cup \Sigma_{2}, \gamma(t)$ adheres to the same component of $\mathrm{Sp}^{+}(2 n)$ (resp. $\left.\mathrm{Sp}^{-}(2 n)\right)$ as $\gamma(0)$ does. This is obvious as long as $\gamma(t) \in \Sigma_{1}$. The only problem could come when $\gamma\left(t_{0}\right) \in \Sigma_{2}$, because in this case $\gamma\left(t_{0}\right)$ seems to adhere to three components of $\Sigma_{0}$ according to proposition A1.1 (compare figure 5). Generically the 2-dimensional eigenspace $V_{1}\left(\gamma\left(t_{0}\right)\right)$ is symplectic. In this case $\gamma\left(t_{0}\right)$ is conjugated to a normal form

$$
\left(\begin{array}{lll}
1 & 0 & \\
0 & 1 & 0 \\
0 & 0 & D
\end{array}\right)
$$

Using the loop

$$
t \in[0,1] \mapsto\left(\begin{array}{ccc}
\cos 2 \pi t & -\sin 2 \pi t & 0 \\
\sin 2 \pi t & \cos 2 \pi t & \\
& 0 & D
\end{array}\right)
$$

we see that, among the three local components of $\Sigma_{0}$ near $\gamma\left(t_{0}\right)$, two of them belong to the same component of $\Sigma_{0}$.

\section{A3. About $\pi_{1}(\operatorname{Sp}(2 n) \backslash \Sigma)$ and co-orientation}

First we recall that, when $A \in \Sigma_{1}$, the eigenvalue 1 has an even multiplicity because its characteristic polynomial is symmetric. Generically on $\Sigma_{1}$ the multiplicity is 2 and a normal form of $A$ up to conjugation is

$$
\left(\begin{array}{lll}
1 & \lambda & 0 \\
0 & 1 & \\
0 & & \Phi
\end{array}\right)
$$


Definition A3.1. We define

$$
\operatorname{sign}(A):=\operatorname{sign}(\lambda \cdot \operatorname{det}(I-\Phi)) .
$$

Proposition A3.2. The sign is well defined and locally constant on $\Sigma_{1}$.

Proof. Here is a simple proof due to N. Depauw and improved by J. Barge. I am pleased to thank both of them for this. Let $e_{1}(A)$ be the unique eigenvector of $A$ with 1 as eigenvalue. Let us consider the endomorphism $M=I-A$. Since $A \in \Sigma_{1}$, the kernel of $M$ is generated by $e_{1}(A)$ and the induced map on the quotient $M^{\prime}: \mathbb{R}^{2 n} / \mathbb{R} e_{1}(A) \rightarrow \mathbb{R}^{2 n}$ is injective. Let $H$ be the $\omega_{0}$-orthogonal of $e_{1}(A)$. As the $\omega_{0}$-product with $e_{1}(A)$ is preserved by $A$, we have $v-A(v) \in H$ for every $v \in \mathbb{R}^{2 n}$. Hence the image of $M^{\prime}$ is a subspace of $H$. Due to the dimensions, $M^{\prime}$ is an isomorphism. The symplectic form allows us to identify the quotient $\mathbb{R}^{2 n} / \mathbb{R} e_{1}(A)$ and $H$. Therefore $M^{\prime}$ can be seen as an isomorphism of $H$. Its determinant $d(A)$ is a well defined continuous function on $\Sigma_{1}$. Using the above notation, we have $d(A)=\lambda \operatorname{det}(I-\Phi)$ when $A$ is generic.

\section{Notation A3.3.}

1) Let us denote $\Sigma_{1}^{ \pm}:=\left\{A \in \Sigma_{1} \mid \operatorname{sign}(A)= \pm\right\}$ and $\Sigma^{ \pm}:=\overline{\Sigma_{1}^{ \pm}}=\Sigma_{1}^{ \pm} \cup \Sigma_{2} \cup$ $\cdots \cup \Sigma_{2 n}$.

2) $\Sigma^{+}$is a hypersurface with singularities of codimension greater than 2 and it is co-oriented, the positive side being $\operatorname{det}(I-A)>0$. Then $\Sigma^{+}$is a homological cycle, having a well defined intersection number $\left[\Sigma^{+}\right] \cdot[\gamma]$ with every free homotopy class $[\gamma]$ of loops in $\operatorname{Sp}(2 n)$. When $\gamma$ is in general position, the intersection number is the number of positive crossings minus the number of negative crossings.

3) For $k \in \mathbb{Z}$, let us define the loop $\gamma_{k}$ by:

$$
t \in[0,1] \mapsto \gamma_{k}(t)=\left(\begin{array}{ccc}
\cos 2 \pi k t & -\sin 2 \pi k t & 0 \\
\sin 2 \pi k t & \cos 2 \pi k t & \\
& 0 & D
\end{array}\right) .
$$

Proposition A3.4. Let $\gamma$ be a loop in $\operatorname{Sp}^{ \pm}(2 n)$. Then $\gamma$ is homotopic to 0 in $\operatorname{Sp}(2 n)$.

Proof. Since $\gamma$ and $\Sigma^{+}$are mutually disjoint we have $\left[\Sigma^{+}\right] \cdot[\gamma]=0$. But we recall from the polar decomposition that $\gamma$ is freely homotopic to the loop $t \mapsto M \cdot \gamma_{k}(t)$ for some $k \in \mathbb{Z}$, where $M$ is any symplectic definite positive symmetric matrix independent of $t$, for instance:

$$
M=\operatorname{diag}\left(\left(\begin{array}{cc}
\xi & 0 \\
0 & \xi^{-1}
\end{array}\right), \cdots,\left(\begin{array}{cc}
\xi & 0 \\
0 & \xi^{-1}
\end{array}\right)\right), \quad \xi>1 .
$$


If $\xi$ is close to 1 , an explicit calculation shows that $M \cdot \gamma_{k}$ crosses $\Sigma^{+} k$ times in the same direction. Thus $\left[\Sigma^{+}\right] \cdot\left[\gamma_{k}\right]= \pm k$, which forces the $k$ associated with the given $\gamma$ to be 0 . (Compare figure 10).

\section{Remark A3.5. Co-orientation of $\Sigma$}

It is clear from the previous discussion that, if the smooth part of $\Sigma$ is globally co-oriented by the sign of $\operatorname{det}(I-A)$, then $\Sigma$ becomes a boundary in homology. For this reason we choose for $\Sigma^{-}$the opposite co-orientation: the positive side of $\Sigma^{-}$will be given by $\operatorname{det}(I-A)<0$. With this co-orientation $\Sigma^{-}$and $\Sigma^{+}$ are homologous in $\operatorname{Sp}(2 n)$. As a consequence we have a clear geometric reason why the Maslov index of a loop is even.

Acknowledgement. I am very grateful to the anonymous referee whose careful reading helped me improving this text. I also thank Nicolas Depauw and JeanClaude Sikorav for several conversations.

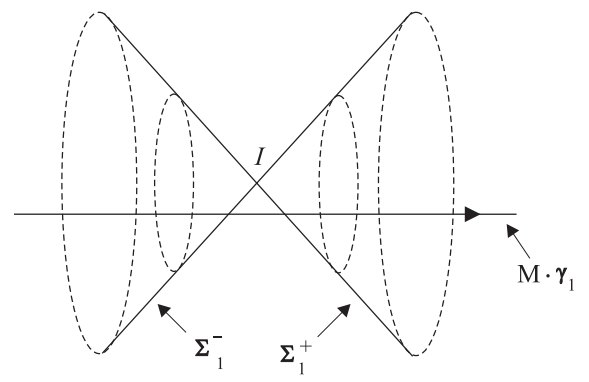

Figure 10

\section{References}

[1] V.I. Arnold, Sur une propriété topologique des applications globalement canoniques de la mécanique classique, C.R. Acad. Sc. Paris 261 (1965), 3719-3722.

[2] V.I. Arnold, Characteristic class entering in quantization conditions, Funct. Anal. Appl. 1 (1967), 1-14. 
[3] V.I. Arnold, Méthodes mathématiques de la mécanique classique, Ed. MIR Moscou 1974, édition française 1976; Mathematical methods of classical mechanics, Springer 1978.

[4] V.I. Arnold, First steps in symplectic topology, Russian Math. Surveys 41-6 (1986), 1-21.

[5] V.I. Arnold, Some remarks on symplectic monodromy of Milnor fibrations, 99-104, in: The Floer memorial volume (H. Hofer, C. Taubes, A. Weinstein, E. Zehnder eds.) Progress in Mathematics, vol. 133, Birkhäuser, 1995.

[6] J. Barge, E. Ghys, Cocycles d'Euler et de Maslov, Math. Ann. 294 (1992), 235-265.

[7] G.D. Birkhoff, Proof of Poincaré's conjecture, Trans. Amer. math. Soc. 14 (1913), 14-22.

[8] B. Booß-Bavnbek, K. P. Wojciechowski, Elliptic boundary problems for Dirac operators, Birkhäuser, 1993.

[9] R. Bott, Lectures on Morse theory, old and new, Bull. Amer. Mat. Soc. 7 (1982), 331-358.

[10] H. Brezis, Analyse fonctionnelle, Théorie et applications, Dunod, Paris, 1999.

[11] M. Chaperon, Une idée du type "géodésique brisée" pour les sytèmes hamiltoniens, C.R. Acad. Sc. Paris 298 (1984), 293-296.

[12] Y. Chekanov, New generalizations of Poincaré's geometric theorem, 113121 in : V.I. Arnold \& al. eds., The Arnold-Gelfand mathematical seminars: geometry and singularity theory, Birkhäuser, 1997.

[13] Y. Chekanov, Differential algebras of Legendrian links, Invent. Math. 150 (2002), 441-483.

[14] C. Conley, E. Zehnder, The Birkhoff-Lewis fixed point theorem and a conjecture of V.I. Arnold, Invent. Math. 73 (1983), 33-49.

[15] C. Conley, E. Zehnder, Morse type index theory for flows and periodic solutions of Hamiltonian equations, Comm. Pure Appl. Math. 37 (1984), 207-253.

[16] S. Dostoglou, D. Salamon, Self dual instantons and holomorphic curves, Annals of Math. 139 (1994), 581-640.

[17] J. Duistermaat, On global action angle coordinates, Comm. Pure and Appl. Math. 33 (1980), 687-708.

[18] Y. Ekeland, Convexity methods in Hamiltonian mechanics, Springer, 1990. 
[19] Y. Eliashberg, A. Givental, H. Hofer, An introduction to Symplectic Field Theory, Geom. Funct. Anal., Special volume, Part II, (2000), 560-673.

[20] J. Etnyre, L. Ng, J. Sabloff, Invariants of Legendrian knots and coherent orientations, J. Symplectic Geom. 1 (2002), 321-367.

[21] A. Floer, Morse theory for Lagrangian intersections, J. Diff. Geometry 28 (1988), 513-547.

[22] A. Floer, The unregularized gradient flow of symplectic action, Comm. Pure Appl. Math. 42 (1989), 775-813.

[23] A. Floer, Witten's complex and infinite dimensional Morse theory, J. Diff. Geometry, 30 (1989), 207-221.

[24] A. Floer, Symplectic fixed points and holomorphic spheres, Comm. Math. Phys. 120 (1989), 575-611.

[25] K. Fukaya, K. Ono, Arnold conjecture and Gromov-Witten invariant, Topology 38 (1999), 933-1048.

[26] R. Gompf, A new construction of symplectic manifolds, Ann. of math. 142 (1995), 527-595.

[27] M. Gromov, Pseudo holomorphic curves in symplectic manifolds, Invent. Math. 82 (1985), 307-347.

[28] H. Hofer, D. Salamon, Floer homology and Novikov rings, 483-524, in: The Floer memorial volume (H. Hofer, C. Taubes, A. Weinstein, E. Zehnder eds.) Progress in Mathematics, vol. 133, Birkhäuser, 1995.

[29] H. Hofer, E. Zehnder, Symplectic invariants and Hamiltonian dynamics, Birkhäuser, 1994.

[30] L. Hörmander, Fourier integral operators I, Acta Mathematica 127 (1971), 79-183.

[31] F. Latour, Existence de 1-formes fermées non singulières dans une classe de cohomologie de de Rham, Pub. Math. IHES 80 (1994), 135-194.

[32] F. Laudenbach, J.-C. Sikorav, Persistance d'intersection avec la section nulle au cours d'une isotopie hamiltonienne dans un fibré cotangent, Invent. Math. 82 (1985), 349-357.

[33] F. Laudenbach, On the Thom-Smale complex, Astérisque 205 (1992), 219233.

[34] P. Le Calvez, Décompositions des difféomorphismes du tore en applications déviant la verticale, 1-120 in: Mémoires Soc. Math. de France 79 (1999). 
[35] G. Liu, G. Tian, Floer homology and Arnold conjecture, J. Diff. Geom. 49 (1998), 1-74.

[36] D. McDuff, D. Salamon, Introduction to symplectic topology, Clarendon Press, Oxford, 1995.

[37] D. McDuff, D. Salamon, J-holomorphic curves and quantum cohomology, Univ. lect. series 6, AMS, 1994.

[38] S.P. Novikov, The Hamiltonian formalism and a multivalued analogue of Morse theory, Russian math. Surveys 37 (1982), 1-56.

[39] P. Ozváth, Z. Szabó, Holomorphic disks and topological invariants for closed three-manifold, arXiv:math.SG/0101206.

[40] P. Ozváth, Z. Szabó, Heegaard Floer homologies and contact structures, arXiv:math.SG/0210127.

[41] H. Poincaré, Sur un théorème de géométrie, Rend. del circ. mat. di Palermo 33 (1912), 375-407; in: CEuvres d'Henri Poincaré, Gauthier-Villars, 1953.

[42] J. Robbin, D. Salamon, The Maslov index for paths, Topology 32 (1993), $827-844$.

[43] J. Robbin, D. Salamon, The spectral flow and the Maslov index, Bull. London Math. Soc. 27 (1995), 1-33.

[44] D. Salamon, Morse theory, the Conley index and Floer homology, Bull. London Math. Soc. 22 (1990), 113-140.

[45] D. Salamon, E. Zehnder, Morse theory for periodic solutions of Hamiltonian systems and the Maslov index, Comm. Pure App. Math. 45 (1992), 1303-1360.

[46] M. Schwarz, Morse Homology, Birkhäuser, 1993.

[47] P. Seidel, The symplectic Floer homology of a Dehn twist, Math. Res. Lett. 3 (1996), 829-834.

[48] P. Seidel, A long exact sequence for symplectic Floer cohomology, Topology 42 (2003), 1003-1063.

[49] J.-C. Sikorav, Homologie associée à une fonctionelle (d'après A. Floer), Séminaire Bourbaki volume 1990/1991, Astérisque 201-202-203 (1991), 115-143.

[50] S. Smale, Morse inequalities for a dynamical system, Bull. Amer. math. Soc. 66 (1960), 43-49.

[51] S. Smale, An infinite dimensional version of Sard's theorem, Amer. J. 87 (1965), 861-866. 
[52] S. Smale, Differentiable dynamical systems, Bull. Amer. Math. Soc. 73 (1967), 747-817.

[53] R. Thom, Sur une décomposition en cellules associée à une fonction sur une variété, C.R. Acad. Sc. Paris 228 (1949), 973-975.

[54] R. Thom, Quelques propriétés globales des variétés différentiables, Comment. Math. Helv. 28 (1954), 17-86.

[55] C. Viterbo, A proof of Weinstein's conjecture in $\mathbb{R}^{2 n}$, Ann. Inst. Henri Poincaré, Analyse Non Linéaire 4 (1987), 337-356.

[56] C. Viterbo, Functors and computations in Floer homology with applications, I, Geom. Funct. Anal. 9 (1999), 985-1033.

[57] A. Weinstein, On the hypotheses of Rabinowitz' periodic orbit theorems, J. Differential Equations 33 (1979), 353-358.

[58] E. Witten, Supersymmetry and Morse Theory, J. of Diff. Geom. 17 (1982), 661-692.

François Laudenbach

Laboratoire Jean Leray, UMR 6629 du CNRS

Faculté des Sciences et Techniques Université de Nantes

F-44322 Nantes cedex 3, France

laudenba@math.univ-nantes.fr 